\title{
Quantum Motion on Shape Space and the Gauge Dependent Emergence of Dynamics and Probability in Absolute Space and Time
}

\author{
Detlef Dürr, ${ }^{1}$ Sheldon Goldstein, ${ }^{2}$ and Nino Zanghí ${ }^{3}$ \\ ${ }^{1}$ Mathematisches Institut der Universität München, Theresienstraße 39, 80333 München, Germany \\ ${ }^{2}$ Departments of Mathematics and Physics, Hill Center, Rutgers University, \\ 110 Frelinghuysen Road, Piscataway, NJ 08854-8019, USA \\ ${ }^{3}$ Dipartimento di Fisica, Università di Genova, \\ Via Dodecaneso 33, 16146 Genova, Italy \\ 8 Istituto Nazionale di Fisica Nucleare (Sezione di Genova)
}

(Dated: August 30, 2019)

\begin{abstract}
Relational formulations of classical mechanics and gravity have been developed by Julian Barbour and collaborators. Crucial to these formulations is the notion of shape space. We indicate here that the metric structure of shape space allows one to straightforwardly define a quantum motion, a Bohmian mechanics, on shape space. We show how this motion gives rise to the more or less familiar theory in absolute space and time. We find that free motion on shape space, when lifted to configuration space, becomes an interacting theory. Many different lifts are possible corresponding in fact to different choices of gauges. Taking the laws of Bohmian mechanics on shape space as physically fundamental, we show how the theory can be statistically analyzed by using conditional wave functions, for subsystems of the universe, represented in terms of absolute space and time.
\end{abstract}

\section{Dedicated to Joel Lebowitz, an invaluable friend and colleague}

\section{CONTENTS}

I. Introduction

II. Shape Space
A. Shapes
B. Metrics on Shape Space
C. Best-Matching
D. Conformal Factors

III. Classical Motion on Shape Space
A. Geodesic Motion
B. Motion in a Potential

IV. Quantum Motion on Shape Space
A. Bohmian Mechanics
B. Bohmian Motion on a Riemannian Manifold
C. Bohmian Motion on Shape Space

V. The Emergence of Absolute Space and Time in the Classical Case
A. Gauge Freedom in the Classical Case
B. Classical Motion in the Newton Gauge
C. Some Remarks on Relational Space and Relational Time
D. Newtonian Gravitation
E. Gauge Freedom, Symmetry Breaking, and Newton's Bucket

\begin{tabular}{l}
2 \\
\\
4 \\
4 \\
4 \\
4 \\
\hline 5 \\
\hline 5 \\
\hline 6 \\
6 \\
\hline 6 \\
\hline
\end{tabular}

VI. The Emergence of Absolute Space and Time in the Quantum Case

A. Gauge Freedom in the Quantum Case 10

B. The Schrödinger Gauge
C. Proofs of the Transitions to the Different Hamiltonians

D. Remarks on the Bohmian Motion in the Various Gauges

14

E. Derivation of the Shape Jacobian 14

F. Computation of the Shape Jacobian 16

G. More Gauge Freedom 17

H. The Canonical Conformal Factor 17

VII. Subsystems 18
A. Conditional Wave Functions
18

B. Subsystems and the Role of Projectivity 18

C. The Emergence of Metrical Time 19

VIII. Probability 19

A. Bohmian Mechanics and Probability 19

B. The Problem of Non-Normalizable Measures

C. The Fundamental Conditional Probability Formula for Evolving Wave Functions

D. The Physical Significance of the Conditional Distribution for Stationary Wave Functions

E. The Association Between Measures on Path Space and on Configuration Space

F. A Conditional Probability Formula for Path Space Measures

G. The Fundamental Conditional Probability Formula for Stationary Wave Functions 24

H. Typicality

IX. Outlook

Acknowledgments 
Appendix: Some facts about second-order partial differential operators

References

\section{INTRODUCTION}

Julian Barbour and Bruno Bertotti, in a very inspiring and influential paper published at the beginning of the eighties [3] (for a recent overview, see [5], [20] and references therein, see also [1]), transformed a long standing philosophical controversy about the nature of space and time into a well-defined physical problem. The philosophical issue dates back to the dispute between Isaac Newton, who favored and argued for the need of an absolute theory of space and time, and Gottfried Wilhelm Leibniz, who insisted upon a relational approach, also defended by Ernst Mach in the 19th century. The physical problem put forward by Barbour and Bertotti can be explained by means of a very elementary and simplified model of the universe.

Suppose we are given the configuration of a universe of $N$ particles. And suppose we translate every particle of the configuration in the same direction by the same amount. From a physical point of view it seems rather natural to take the relational point of view that the two configurations of the universe so obtained are physically equivalent or identical. Similarly for any rotation. Going one step further, one regards two configurations of the universe differing only by a dilation, i.e. by a uniform expansion or contraction, as representing in fact the same physical state of the universe. The space of all genuinely physically different possible configurations so obtained - taking into account translations, rotations, and dilations - is usually called shape-space. The name shape-space is indeed natural: only the shape of a configuration of particles is relevant, not its position or orientation or overall size.

Given a kinematics based on shapes, the next question to be addressed is that of their dynamics. In their seminal paper, Barbour and Bertotti proposed a dynamical principle based on what they called the intrinsic derivative and Barbour now calls best matching, which allows one to compare two shapes intrinsically, without any reference to the external space in which the particles are embedded. While the intrinsic comparison of shapes is compatible with positing an absolute Newtonian time as in classical mechanics, it naturally leads to a relational notion of time in which global changes of speed of the history of the universe give physically equivalent representations. Then the dynamics can be reduced to geometry in the following sense: a history of the universe is just a curve in shape space without any reference to a special parametrization of the curve given by absolute Newtonian time.

The goal of the present paper is to extend the foregoing to the quantum case. We shall do this by considering the toy model mentioned above in which the universe is modelled as an $N$-particle system. This will suffice to highlight the general feature of a relational quantum theory of the universe. However, we shall do so not by appealing to standard quantization schemes (see e.g., 2], [14]), but by relying on the precise formulation of quantum theory provided by Bohmian mechanics [7, 9, 11, 12. Steps in this direction have been taken by Vassallo and Ip 22] and by Koslowski [17.

Bohmian mechanics is a theory providing a description of reality, compatible with all of the quantum formalism, but free of any reference to observables or observers. In Bohmian mechanics a system of particles is described in part by its wave function, evolving according to Schrödinger's equation, the central equation of quantum theory. However, the wave function provides only a partial description of the system. This description is completed by the specification of the actual positions of the particles. The latter evolve according to the "guiding equation," which expresses the velocities of the particles in terms of the wave function. Thus in Bohmian mechanics the configuration of a system of particles evolves via a deterministic motion choreographed by the wave function.

Given the primary role of configurations, as opposed to operators and canonical quantization relations, it should not come as a surprise that Bohmian mechanics can be very easily formulated on shape space: a wave function on shape space will govern the motion of a shape according to a guiding law analogous to the one of standard Bohmian mechanics. And to express the guiding law, as well as to write down Schrödinger's equation on shape space, all one needs is a metric on shape space.

Surprisingly (or maybe not), the properties of metrics on shape space have been investigated by applied mathematicians before the paper of Barbour and Bertotti, and for completly different reasons. What in physics is a configuration of $N$ particles, in statistics is a set of data, and data analysis often requires that all information in a data set about its location, scale, and orientation be removed, so that the information that remains provides an intrinsic description of the shape of the data. Indeed, the name "shape space" is due to the mathematicians that have been working on these problems of data analysis. In particular David G. Kendall, whose early work on shape space dates back to the 1970s, was concerned with shape in archaeology and astronomy and also considered the motion of shapes formed by independent Brownian particles [15], while Fred Bookstein at about the same time began to study shape-theoretic problems in the particular context of zoology. Both recognized that the space of shapes can be represented by Riemannian manifolds (see 16. 21] for more background). We shall briefly review how to construct a metric on shape space in Sect. II]

Not only in Bohmian mechanics, but also in the classical theory of Barbour and Bertotti, a metric on shape space plays a pivotal role in the formulation of the theory. Indeed, it turns out that Barbour's best-matching 
principle is equivalent to a characterization of the dynamics as geodesic motion in shape space. Though this fact was acknowledged by the authors in their original paper (and also in more recent publications by Barbour and collaborators), we think that sufficient emphasis has not been given to it. Usually, classical motion on shape space is characterized by means of Lagrangian or Hamiltonian formulations with constraints (see, e.g., 4). While we agree that such methods of analytical mechanics could be useful in the analysis of the theory, we think that they obscure the geometrical structure of the theory. So in Sect. III we shall provide a self-contained presentation of the classical theory by emphasizing its geometrical content, in particular that the dynamics of shapes (even in presence of interactions) is geodesic motion on shape space. In Sect. [IV] we shall develop the Bohmian theory of motion and highlight the similarities and differences between the classical case and the quantum case.

An important point that we think has not been given sufficient emphasis is that the fundamental formulations of the theories - classical or quantum - are in shape space. And when the theories are formulated in shape space, one should consider first the simplest ones, namely the "free" theories based only on the geometrical structures provided by the metric, without invoking any potential. This is in contrast with theories formulated in absolute space, for which free theories can't begin to account for the experimental data. It is then natural to ask: when we represent the theories in absolute space, what form do the laws of motion take? Is the representation unique or are there various representations yielding different looking laws of motion, some unfamiliar and some more or less familiar? Moreover do interacting theories emerge with nontrivial interactions, although in shape space the motion is free?

To answer these questions it is helpful to represent absolute configuration space in geometrical terms as a fiber bundle, with shape space as base manifold and the fibers generated by the similarity group, i.e, by translations, rotations and dilations, which acting on configurations yields, from a relational point of view, physically equivalent states. A representation in absolute configuration space of the motion in shape space is then given by a "lift" of the motion from the base into the fibers.

Such lifts can rightly be called gauges. In the classical case it turns out that in some gauges the law looks unfamiliar but there is (at least) one gauge in which, after performing a time change (representing indeed another gauge freedom when also time is seen as relational), the law of motion is Newtonian with a potential appearing. The potential depends on the choice of the invariant metric (invariant under the action of the similarity group) in absolute configuration space, which we introduce in Sect. II], where various possibilities for invariant metrics are given. The classical case is dealt with in Sect. $\mathrm{V}$.

More or less the same is true for the quantum case, where however the gauge yielding ordinary Bohmian mechanics in absolute configuration space - which we call the Schrödinger gauge - emerges only for a stationary, i.e. time-independent, wave function (such as with the Wheeler-DeWitt equation) on shape space. This again is in line with regarding time as being relational, with an external absolute time playing no physical role.

Also here, while the fundamental physics is given by a free Bohmian dynamics in shape space, in the Schrödinger gauge potential terms appear. One potential term is determined by the scalar curvature induced by the invariant metric on absolute configuration space. Another potential term arises from the gauge freedom we have to lift the Laplace-Beltrami operator from shape space to absolute configuration space, where an extra gauge freedom arises from allowing transformations of the lifted wave function. To see the Schrödinger gauge arise, we invoke some mathematical facts from differential geometry. The details are in Sect. VI

Regarding the motion in shape space as physically fundamental, we may well conclude from Sect.s $\mathrm{V}$ and VI that the gauge freedom forces us to recognize that what we have traditionally regarded as fundamental might in fact be imposed by us through our choice of gauge. This gauge freedom thus imparts a somewhat Kantian aspect to physical theory.

We next turn to the issue of probability, given by the quantum equilibrium measure $|\Psi|^{2}$ on shape space. In assessing the relationship between probability on shape space and the usual Born-rule probabilities on absolute configuration space (associated with the natural lifts of the shape space dynamics to absolute space), we encounter several problems. First of all, since wave functions lifted from shape space are translation and scaling invariant, they fail to be normalizable. Another source of non-normalizability is the transition to the Schrödinger gauge. For this gauge to be viable, as stated earlier, the wave function must be time-independent, and such wave functions typically fail, as with those of the WheelerDeWitt equation, to be normalizable.

So what could the associated non-normalizable "probabilities" physically mean? Moreover, the physical meaning of these measures would be obscure even if they were normalizable, since the absolute space degrees of freedom that transcend the relational ones are not observable, and the configuration $Q_{t}$ of the universe at "time $t$," whose distribution is supposed to be given by the Born rule, is, as we argue, not physically meaningful.

We address these questions in Sect. VIII in which we examine what should be physically and observationally meaningful, and find that the relevant probabilities for these are in fact given by a fundamental conditional probability formula (see [11] for its meaning in the familiar Bohmian mechanics), as normalized conditional probabilities arising from the non-normalizable quantum equilibrium measure on absolute configuration space. For this we use the notion of the wave function of a subsystem of shape space, a somewhat tricky business that is dealt with in Sect. VII.

We find in fact, somewhat to our surprise, that the 


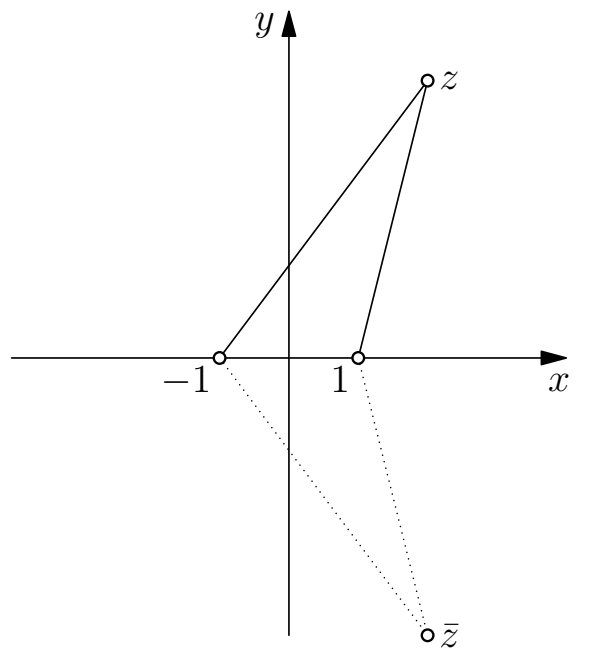

FIG. 1. Representation of the shape space of 3 particles in terms of point $z$ in the complex upper half plane. Note that the complex conjugate $\bar{z}$ represents the same triangle since it can be obtained from that of $z$ by a rotation in 3-dimensional Euclidean space.

non-normalizability of the wave function of the universe of quantum cosmology is, from a relational Bohmian perspective, a virtue rather than a vice.

\section{SHAPE SPACE}

\section{A. Shapes}

The totality of configurations $\boldsymbol{q}=\left(\overrightarrow{\mathrm{q}}_{1}, \ldots, \overrightarrow{\mathrm{q}}_{N}\right)$ of $N$ points in Euclidean three-dimensional space forms the configuration space $\mathbf{Q}=\{\boldsymbol{q}\}=\mathbb{R}^{3 N}$ of an $N$-particle system. We shall call $\mathbb{Q}$ the absolute configuration space. On 2 act naturally the similarity transformations of Euclidean space, namely rotations, translations and dilations, since each of them acts naturally on each component of the configuration vector. The totality of such transformations form the group $G$ of similarity transformations of Euclidean space. Since the shape of a configuration is "what is left" when the effects associated with rotations, translations and dilations are filtered away, the totality of shapes, i.e., the shape space, is the quotient space $2 \equiv 2 / G$, the set of equivalence classes with respect to the equivalence relations provided by the similarity transformations of Euclidean space.

As such, shape space is not in general a manifold. To transform it into a manifold some massaging is needed (e.g., by excluding from 2 coincidence points and collinear configurations), but we shall not enter into this ${ }^{1}$ Here, we shall assume that the appropriate massaging of

\footnotetext{
${ }^{1}$ For more details on this issue, see, e.g., [18] and reference therein.
}

2 has been performed and that 2 is a manifold. Since the group of similarity transformations has dimension 7 ( 3 for rotations +3 for translations +1 for dilations), the dimension of $\mathcal{Q} \equiv \mathcal{Q} / G$ is $3 N-7$.

For $N=1$ and $N=2$ shape space is trivial (it contains just a single point). $N=3$ corresponds to the simplest not trivial shape space; it has dimension $3 \times 3-7=2$. It is worthwhile to give some details about this latter case. Three points in Euclidean space form a triangle, so shape space is the space of all triangle shapes, with "triangle shape" meaning now what is usually meant in elementary Euclidean geometry. A nice representation of this space is in terms of points in the complex plane (called Bookstein-coordinates in 21]). On the real axis, fix two points, say -1 and 1 , and put them in correspondence with two vertices of the triangle. Then the third vertex is in one-to-one correspondence with a complex number in the upper half plane, as shown in Fig. 1. Note that the triangles in the lower half plane are equivalent to those in the upper half plane by a suitable rotation in three dimensions. The real axis is the boundary of the manifold and its points represent degenerate collinear triangles. The point at infinity represents the degenerate triangular shape with two coinciding vertices. So the space of triangle shapes (allowing two coincident vertices but not three) can be put in correspondence with the the extended half upper complex plane, which, by stereographic projection, is topologically equivalent to a hemisphere. For $N>3$ the topological structure is more complicated (see, e.g., [18]).

\section{B. Metrics on Shape Space}

Topology, of course, does not fix a metric. A metric should provide more, namely a natural notion of distance on 2 . And since each point in 2 represents a class of configurations of $N$ particles related by a similarity transformation, the distance between two elements of $\mathcal{Q}$ induced by the metric should not recognize any absolute configurational difference due to an overall translation, or rotation, or dilation. In other words, it should provide a measure of the intrinsic difference between two absolute configurations (that is, not involving any consideration regarding how such configurations are embedded in Euclidean space).

Although the construction of such a metric is well known in the mathematical literature on random shapes [18, we prefer to give a self-contained presentation more suited for the physical applications. The bottom line is this: a metric on absolute configuration space 2 that is invariant under the group $G$ of similarity transformations of Euclidean space, given by a suitable "conformal factor" (to be explained below), defines canonically a metric on shape space 2 .

To understand why this is so, observe first that absolute configuration space $\mathbf{Q}$ can be regarded as a fiber bundle with each fiber being homeomorphic to $G$ and 2 


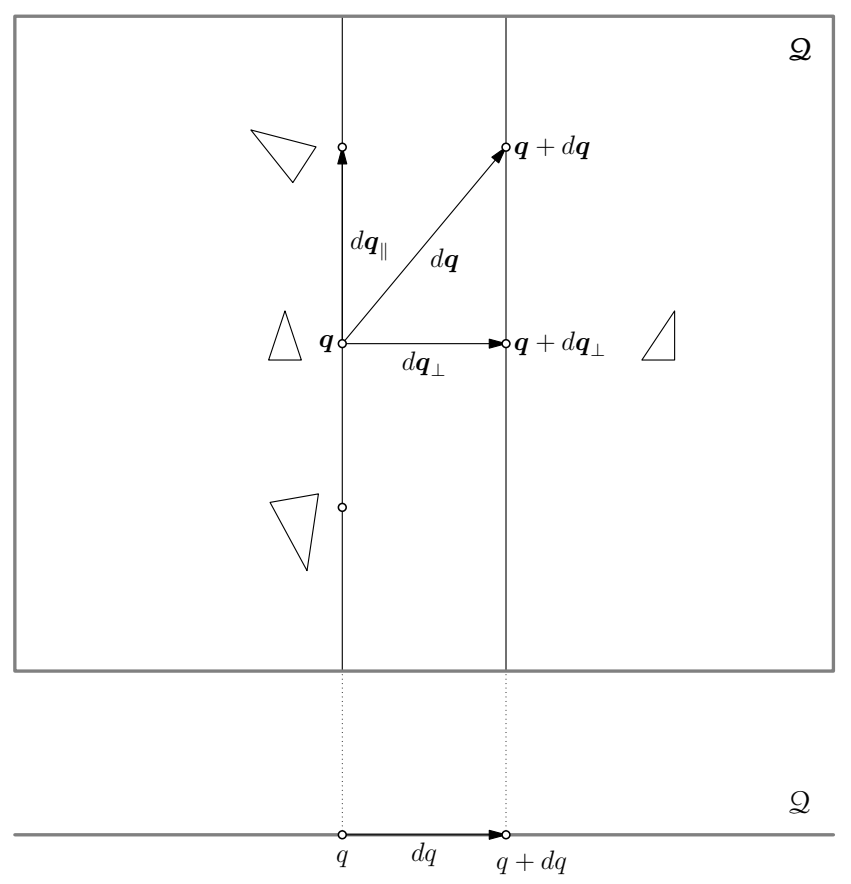

FIG. 2. Absolute configuration space $\mathcal{2}$ and shape space 2 (for a system of three particles). The fiber above shape $q$ consists of absolute configurations differing by a similarity transformation of Euclidean space and thus representing the same shape $q$. Real change of shape occurs only by a displacement to a neighboring fiber $q+d q$. Only the orthogonal component $d \boldsymbol{q}_{\perp}$ of $d \boldsymbol{q}$ represents real change, while the vertical displacement $d \boldsymbol{q}_{\|}$does not contribute; $\boldsymbol{q}+d \boldsymbol{q}_{\perp}$ is the absolute configuration in the fiber above $q+d q$ closest to $q$ in the sense of the $g_{B}$-distance (best matching).

being its base space (see Fig. 2). So, if $g$ is a metric invariant under any element of $G$, the tangent vectors at each point $\boldsymbol{q} \in \mathbf{Q}$ are naturally split into "vertical" and "horizontal," where by "naturally" we mean that the splitting itself is invariant under the action of $G$. The vertical ones correspond to (infinitesimal) displacements along the fiber through $\boldsymbol{q}$ and the horizontal ones are those that are orthogonal to the fiber, i.e., to the vertical ones, according to the relation of orthogonality defined by $g$. More precisely, if $d \boldsymbol{q}$ is an infinitesimal displacement at $\boldsymbol{q}$, we have

$$
d \boldsymbol{q}=d \boldsymbol{q}_{\|}+d \boldsymbol{q}_{\perp} \quad \text { with } \quad g\left(d \boldsymbol{q}_{\|}, d \boldsymbol{q}_{\perp}\right)=0
$$

(see Fig. 2), with $d \boldsymbol{q}_{\|}$vertical and $d \boldsymbol{q}_{\perp}$ horizontal.

The corresponding Riemannian metric on $\mathcal{Q}$ is defined as follows. Let $q$ be a shape, $\boldsymbol{q}$ be any absolute configuration in the fiber above $q$, and $d \boldsymbol{q}$ be any displacement at $\boldsymbol{q}$. Since $g$ is invariant under the group $G$, the length of $d \boldsymbol{q}_{\perp}$ has the same value for all absolute configurations $\boldsymbol{q}$ above $q$. Then we may set the length of $d q$ equal to that of $d \boldsymbol{q}_{\perp}$ and hence obtain the Riemannian metric $g_{B}$ on 2

$$
g_{B}(d q, d q)=g\left(d \boldsymbol{q}_{\perp}, d \boldsymbol{q}_{\perp}\right)
$$

The subscript $B$ stands for Barbour and Bertotti (as well as base and best matching, see below).

We shall now outline how to construct an invariant metric on $\mathbf{2}$. Let $g_{e}$ be the mass-weighted Euclidean metric on $\mathcal{Q}$ with positive weights $m_{\alpha}, \alpha=1, \ldots, N$, (the masses of the particles), in particle coordinates $\boldsymbol{q}=$ $\left(\overrightarrow{\mathrm{q}}_{1}, \ldots, \overrightarrow{\mathrm{q}}_{\alpha}, \ldots, \overrightarrow{\mathrm{q}}_{N}\right)$ given by

$$
d s^{2}=\sum_{\alpha=1}^{N} m_{\alpha} d \overrightarrow{\mathrm{q}}_{\alpha} \cdot d \overrightarrow{\mathrm{q}}_{\alpha},
$$

i.e., with $\left[g_{e}\right]_{i j}=m_{\alpha_{i}} \delta_{i j}$, where the $i$-th component refers to the $\alpha_{i}$-th particle. The corresponding line element is

$$
|d \boldsymbol{q}|=\sqrt{\sum_{\alpha=1}^{N} m_{\alpha} d \overrightarrow{\mathrm{q}}_{\alpha} \cdot d \overrightarrow{\mathrm{q}}_{\alpha}}
$$

The metric defined by (2) is invariant under rotations and translations, but not under a dilation $\boldsymbol{q} \rightarrow \lambda \boldsymbol{q}$, where $\lambda$ is a positive constant. Invariance under dilations is achieved by multiplying $|d \boldsymbol{q}|^{2}$ by a scalar function $f(\boldsymbol{q})$ that is invariant under rotations and translations and is homogeneous of degree -2 . We call $f$ the conformal factor. So, for any choice of $f$,

$$
g=f g_{e}, \quad \text { i.e, } \quad g(d \boldsymbol{q}, d \boldsymbol{q})=f(\boldsymbol{q})|d \boldsymbol{q}|^{2}
$$

is an invariant metric on $\mathcal{Q}$, yielding the metric on shape space

$$
g_{B}(d q, d q)=f(\boldsymbol{q})\left|d \boldsymbol{q}_{\perp}\right|^{2}
$$

For the associated line element we shall write

$$
d s=|d q|=\sqrt{g_{B}(d q, d q)}=\sqrt{f(\boldsymbol{q})}\left|d \boldsymbol{q}_{\perp}\right| .
$$

\section{Best-Matching}

The distance on 2 induced by $g_{B}$ is exactly the one resulting from applying Barbour's best matching procedure. Consider two infinitesimally close shapes, $q$ and $q+d q$, and let $\boldsymbol{q}$ be any absolute representative of $q$, i.e., any point in the fiber above $q$. The $g_{B}$-distance between these shapes is then given by the $g$-length of the vector $d \boldsymbol{q}$ such that (i) $d \boldsymbol{q}$ is orthogonal to the fiber above $q$ and (ii) $\boldsymbol{q}+d \boldsymbol{q}$ is an absolute representative of $q+d q$. It follows that $\boldsymbol{q}+d \boldsymbol{q}$ is the absolute configuration closest to $\boldsymbol{q}$ in the fiber above $q+d q$. Thus the $g_{B}$-distance is the "best matching" distance.

\section{Conformal Factors}

Many choices of conformal factors are possible. One that was originally suggested by Barbour and Bertotti 
is ${ }^{2}$

$$
f(\boldsymbol{q})=f_{a}(\boldsymbol{q}) \equiv\left(\sum_{\alpha<\beta} \frac{m_{\alpha} m_{\beta}}{\left|\overrightarrow{\mathrm{q}}_{\alpha}-\overrightarrow{\mathrm{q}}_{\beta}\right|}\right)^{2} .
$$

Another example is

$$
f(\boldsymbol{q})=f_{b}(\boldsymbol{q}) \equiv \mathrm{L}^{-2},
$$

where

$$
\mathrm{L}^{2}=\sum_{\alpha} m_{\alpha}{\overrightarrow{q_{\alpha}}}^{2}=\frac{1}{\sum_{\alpha} m_{\alpha}} \sum_{\alpha<\beta} m_{\alpha} m_{\beta}\left|\overrightarrow{\mathrm{q}}_{\alpha}-\overrightarrow{\mathrm{q}}_{\beta}\right|^{2}
$$

with $\vec{q}_{\alpha}=\vec{q}_{\alpha}-\vec{q}_{\mathrm{cm}}$, the coordinates relative to the center of mass

$$
\overrightarrow{\mathrm{q}}_{\mathrm{cm}}=\frac{\sum_{\alpha} m_{\alpha} \overrightarrow{\mathrm{q}}_{\alpha}}{\sum_{\alpha} m_{\alpha}}
$$

$I \equiv \mathrm{L}^{2}$ is sometimes called (but the terminology is not universal) the moment of inertia of the configuration $\boldsymbol{q}$ about its center of mass. This quantity is half the trace of the moment of inertia tensor $\mathrm{M}$,

$$
\mathrm{L}^{2}=\frac{1}{2} \operatorname{Tr} \mathrm{M}
$$

We recall that $M=M(\boldsymbol{q})$, the tensor of inertia of the configuration $\boldsymbol{q}$ about any orthogonal cartesian system $x, y, z$ with origin at the center of mass of the configuration $\boldsymbol{q}$, has matrix elements given by the standard formula

$$
M_{i j}=\sum_{\alpha=1}^{N} m_{\alpha}\left(\rho_{\alpha}^{2} \delta_{i j}-\rho_{\alpha i} \rho_{\alpha j}\right),
$$

where $i, j=x, y, z, \rho_{\alpha x} \equiv x_{\alpha}, \rho_{\alpha y} \equiv y_{\alpha}, \rho_{\alpha z} \equiv z_{\alpha}$, and $\rho_{\alpha}^{2}=x_{\alpha}^{2}+y_{\alpha}^{2}+z_{\alpha}^{2}$.

A choice of conformal factor that has not been considered in the literature is

$$
f(\boldsymbol{q})=f_{c}(\boldsymbol{q}) \equiv \mathrm{L}^{-\frac{8}{7}}(\operatorname{det} \mathrm{M})^{-\frac{1}{7}} .
$$

Since $\operatorname{det} \mathrm{M}$ scales as $\mathrm{L}^{6}, f(\boldsymbol{q})$ given by 13 scales as it should, namely, as $\mathrm{L}^{-2}$. Though at first glance this choice does not seem natural, it is in fact so naturalonce the motion of shapes is analyzed from a quantum perspective, see Sect. VIH - that we shall call $f_{c}$ the canonical conformal factor.

Finally, we give other two examples:

$$
\begin{aligned}
& f(\boldsymbol{q})=f_{d}(\boldsymbol{q}) \equiv \sum_{\alpha<\beta} \frac{m_{\alpha} m_{\beta}}{\left|\overrightarrow{\mathrm{q}}_{\alpha}-\overrightarrow{\mathrm{q}}_{\beta}\right|^{2}} \\
& f(\boldsymbol{q})=f_{g}(\boldsymbol{q}) \equiv \mathrm{L}^{-1} \sum_{\alpha<\beta} \frac{m_{\alpha} m_{\beta}}{\left|\overrightarrow{\mathrm{q}}_{\alpha}-\overrightarrow{\mathrm{q}}_{\beta}\right|} .
\end{aligned}
$$

The first one corresponds to a natural modification of the Newtonian gravitational potential and the second, discussed in 4, corresponds to a dynamics very close to that of Newtonian gravity (see Sect. $\mathrm{VB}$ and Sect. $\mathrm{VD}$.

\footnotetext{
${ }^{2}$ Here and in the following examples the conformal factors are modulo dimensional factors.
}

\section{CLASSICAL MOTION ON SHAPE SPACE}

\section{A. Geodesic Motion}

The metric $g_{B}$ on shape space directly yields a law of free motion on shape space, that is, geodesic motion with constant speed. More explicitly, this is the motion $Q=$ $Q(t)$ at constant speed along the path that minimizes the length

$$
\int_{q_{1}}^{q_{2}}|d q|=\int_{\boldsymbol{q}_{1}}^{\boldsymbol{q}_{2}} \sqrt{f(\boldsymbol{q})}\left|d \boldsymbol{q}_{\perp}\right|
$$

over all possible paths connecting two shapes $q_{1}$ and $q_{2}$ (if they are sufficiently close). Note that the variational problem determines only the path of the motion, but not the motion in time.

Equivalently, a geodesic motion $Q=Q(t)$ is a motion that parallel-transports its own tangent vector, so

$$
D_{\dot{Q}(t)} \dot{Q}(t)=0,
$$

where $D_{\dot{Q}(t)}$ is the covariant derivative with respect to the metric $g_{B}$ along the curve $Q=Q(t)$. Given the initial conditions $Q(0)$ and $\dot{Q}(0)$, the motion will run at constant speed $v=|\dot{Q}(0)|$.

\section{B. Motion in a Potential}

Motion under the effect of the potential $\mathscr{V}=\mathscr{V}(q)$ is given by the obvious modification of (17), namely Newton's equation

$$
D_{\dot{Q}(t)} \dot{Q}(t)=-\nabla_{g_{B}} \mathscr{V}(Q),
$$

where $\nabla_{g_{B}}$ is the gradient with respect to the metric $g_{B}$. This is equivalent to a characterization of the motion in terms of the Lagrangian

$$
L=\frac{1}{2} g_{B}\left(\frac{d q}{d t}, \frac{d q}{d t}\right)-\mathscr{V}(q)=\frac{1}{2}\left|\frac{d q}{d t}\right|^{2}-\mathscr{V}(q) .
$$

\section{QUANTUM MOTION ON SHAPE SPACE}

\section{A. Bohmian Mechanics}

Various quantization schemes have been put forward in order to provide a quantum theory of motion on shape space; for a thorough overview, see [1]. These schemes are mostly based on Dirac quantization of classical constrained systems or on Feynman path integration [14. We shall follow here a novel approach based on Bohmian mechanics. Bohmian mechanics is a completely deterministic - but distinctly non-Newtonian - theory of particles in motion, with the wave function itself guiding this motion. We shall explain below how this theory can be 
naturally formulated on shape space, after a brief review of the main features of the theory.

Bohmian mechanics is the minimal completion of Schrödinger's equation, for a non-relativistic system of particles, to a theory describing a genuine motion of particles. For Bohmian mechanics the state of a system of $N$ particles is described by its wave function $\Psi=\Psi\left(\overrightarrow{\mathrm{q}}_{1}, \ldots, \overrightarrow{\mathrm{q}}_{N}\right)=\Psi(\boldsymbol{q})$, a complex- (or spinor-) valued function on the space of possible configurations $\boldsymbol{q}$ of the system, together with its actual configuration $\boldsymbol{Q}$ defined by the actual positions $\vec{Q}_{1}, \ldots, \vec{Q}_{N}$ of its particles. The theory is then defined by two evolution laws. One is Schrödinger's equation

$$
i \hbar \frac{\partial \Psi}{\partial t}=H \Psi
$$

for $\Psi=\Psi_{t}$, the wave function at time $t$, where $H$ is the non-relativistic (Schrödinger) Hamiltonian, containing the masses $m_{k}, k=1, \ldots, N$, of the particles and a potential energy term $V$. For spinless particles, it is of the form

$$
H=-\sum_{\alpha=1}^{N} \frac{\hbar^{2}}{2 m_{\alpha}} \vec{\nabla}_{\alpha}^{2}+V
$$

where $\vec{\nabla}_{\alpha}=\frac{\partial}{\partial \overrightarrow{\mathbf{q}}_{\alpha}}$ is the gradient with respect to the position of the $\alpha$-th particle. The other law is the the guiding law, which, for spinless particles, is given by the equation 3

$$
\frac{d \overrightarrow{\mathrm{Q}}_{\alpha}}{d t}=\frac{\hbar}{m_{\alpha}} \operatorname{Im} \frac{\vec{\nabla}_{\alpha} \Psi}{\Psi}\left(\overrightarrow{\mathrm{Q}}_{1}, \ldots, \overrightarrow{\mathrm{Q}}_{N}\right)
$$

for $\boldsymbol{Q}=\boldsymbol{Q}(t)$, the configuration at time $t$. For an $N$ particle system these two equations, together with the detailed specification of the Hamiltonian $H$, completely define the Bohmian motion of the system. For sake of simplicity, we shall consider here just Bohmian mechanics for spinless particles, with Hamiltonian (21) and guiding law (22). For more details on the formulation of Bohmian mechanics for particles with spin or other internal degrees of freedom, see 12 .

While the formulation of Bohmian mechanics does not involve the notion of quantum observables, as given by self-adjoint operators - so that its relationship to the quantum formalism may at first appear somewhat obscure - it can in fact be shown that Bohmian mechanics not only accounts for quantum phenomena, but also

3 The general form of the guiding equation is

$$
\frac{d \overrightarrow{\mathbf{Q}}_{\alpha}}{d t}=\frac{\hbar}{m_{\alpha}} \operatorname{Im} \frac{\Psi^{*} \vec{\nabla}_{\alpha} \Psi}{\Psi^{*} \Psi}\left(\overrightarrow{\mathrm{Q}}_{1}, \ldots, \overrightarrow{\mathrm{Q}}_{N}\right) .
$$

If $\Psi$ is spinor-valued, the products in numerator and denominator should be understood as scalar products. If external magnetic fields are present, the gradient should be understood as the covariant derivative, involving the vector potential. embodies the quantum formalism itself as the very expression of its empirical import [12, Ch.2 and 3].

It is worth noting that the guiding equation 22 is intimately connected with the de Broglie relation $\vec{p}=\hbar \vec{k}$, proposed by de Broglie in late 1923, the consideration of which quickly led Schrödinger to the discovery of his wave equation. The de Broglie relation connects a particle property, momentum $\vec{p}=m \vec{v}$, to a wave property, the wave vector $\vec{k}$ of a plane wave $\Psi(\vec{q})=e^{i \vec{k} \cdot \vec{q}}$. From this one can easily guess the guiding equation as the simplest possibility for an equation of motion for $\boldsymbol{Q}$ for the case of a general wave function $\Psi$.

\section{B. Bohmian Motion on a Riemannian Manifold}

Note that, given $V$, the Bohmian mechanics defined by equations (20), (21), and (22) depends only upon the Riemannian structure $g=g_{e}$ given by (2). In terms of this Riemannian structure, the evolution equations (21) and 22 become

$$
\begin{aligned}
\frac{d Q}{d t} & =\hbar \operatorname{Im} \frac{\nabla_{g} \Psi}{\Psi} \\
i \hbar \frac{\partial \Psi}{\partial t} & =-\frac{\hbar^{2}}{2} \Delta_{g} \Psi+V \Psi,
\end{aligned}
$$

where $\Delta_{g}$ and $\nabla_{g}$ are, respectively, the Laplace-Beltrami operator and the gradient on the configuration space equipped with this Riemannian structure. But there is nothing special about this particular Riemannian structure. Indeed, equations (23) and (24) as such hold very generally on any Riemannian manifold. Thus, the formulation of a Bohmian dynamics on a Riemannian manifold requires only as basic ingredients the differentiable and metric structure of the manifold.

\section{Bohmian Motion on Shape Space}

Equations 23 and 24 define immediately Bohmian motion on shape space with Riemannian metric $g=g_{B}$ as the motion on shape space given by the evolution equations

$$
\begin{aligned}
\frac{d Q}{d t} & =\hbar \operatorname{Im} \frac{\nabla_{B} \Psi}{\Psi} \\
i \hbar \frac{\partial \Psi}{\partial t} & =-\frac{\hbar^{2}}{2} \Delta_{B} \Psi+\mathscr{V} \Psi,
\end{aligned}
$$

where $\Delta_{B}$ and $\nabla_{B}$ are, respectively, the LaplaceBeltrami operator and the gradient on the configuration space equipped with the Riemannian metric (5). This is all there is to say about the formulation of Bohmian mechanics on shape space. (This should be contrasted with more involved approaches as in, e.g., [22.) 


\section{THE EMERGENCE OF ABSOLUTE SPACE AND TIME IN THE CLASSICAL CASE}

\section{A. Gauge Freedom in the Classical Case}

Given classical motion in shape space, there is a huge host of motions in absolute space that are compatible with it, the only constraint being that they should project down to free motion, or the motion (18), in shape space. This freedom of choice is analogous to gauge freedom in gauge theories. Some choices are however more natural than others, as we shall discuss below.

\section{B. Classical Motion in the Newton Gauge}

A very natural choice of a motion in absolute configuration space is the horizontal lift of a motion $Q=Q(t)$ in shape space, that is, a motion $\boldsymbol{Q}=\boldsymbol{Q}(t)$ in absolute configuration space that starts at some point $\boldsymbol{q}_{1}$ on the fiber above $q_{1}$ and is horizontal, i.e., the infinitesimal displacements $d \boldsymbol{Q}$ are all horizontal. (Note that the final point $\boldsymbol{q}_{2}$ in the fiber above $q_{2}$ is then uniquely determined.) We call this choice the invariant gauge.

We shall assume $\mathscr{V}=04$ Then the motion in the invariant gauge is geodesic motion with respect to the invariant metric (which explains the terminology). To see this, observe that it follows from (16) that the length of a horizontal lift of a path in shape space is given by

$$
\int_{\boldsymbol{q}_{1}}^{\boldsymbol{q}_{2}} \sqrt{f(\boldsymbol{q})}\left|d \boldsymbol{q}_{\perp}\right|=\int_{\boldsymbol{q}_{1}}^{\boldsymbol{q}_{2}} \sqrt{f(\boldsymbol{q})}|d \boldsymbol{q}|,
$$

where the equality follows from horizontality of the path. So, the path of a horizontal lifted motion $\boldsymbol{Q}=\boldsymbol{Q}(t)$ has minimal length over all horizontal paths connecting $\boldsymbol{q}_{1}$ and $\boldsymbol{q}_{2}$, but since any non horizontal path has a greater length, $\boldsymbol{Q}(t)$ also minimizes the right hand side of (27) over all paths connecting $\boldsymbol{q}_{1}$ and $\boldsymbol{q}_{2}$.

We shall now show that by a suitable change of speed, we get to another gauge that we shall call the Newton gauge, a gauge in which the motion is Newtonian, i.e., it satisfies Newton's equation $F=m a$ for suitable $F$. To establish this, we first observe that the right hand side of 27) is of the form

$$
\int_{\boldsymbol{q}_{1}}^{\boldsymbol{q}_{2}} \sqrt{E-V}|d \boldsymbol{q}|
$$

for $E=0$ and $V(\boldsymbol{q})=-f(\boldsymbol{q})$. According to the Jacobi principle, 28 is minimized by the path of a Newtonian motion $\boldsymbol{Q}^{\prime}=\boldsymbol{Q}^{\prime}(t)$ in a potential $V$ and total energy

$$
E=\frac{1}{2}\left|\frac{d \boldsymbol{Q}^{\prime}}{d t}\right|^{2}+V=0
$$

\footnotetext{
4 Our goal is to show that the simplest dynamics on shape space leads to a nontrivial dynamics in a suitable gauge. The case $\mathscr{V} \neq 0$ will be considered in the next subsection.
}

Thus the path of a lifted motion $\boldsymbol{Q}=\boldsymbol{Q}(t)$ is the same as that of a Newtonian motion, but its speed along the path is different: according to 29 the speed of the Newtonian motion is

$$
\left|\frac{d \boldsymbol{Q}^{\prime}}{d t}\right|=\sqrt{2(E-V)}=\sqrt{2 f},
$$

while according to (6) the speed of the lifted motion is

$$
\left|\frac{d \boldsymbol{Q}}{d t}\right|=\frac{1}{\sqrt{f}}\left|\frac{d q}{d t}\right|=\frac{v}{\sqrt{f}},
$$

with $v$ the constant speed of the motion on shape space. So the two motions are different. But suppose we allow for a change of the flow of time and replace $t$ with a new time variable $t^{\prime}$ in such a way that the speed of the lifted motion with respect to this new time variable equals the Newtonian speed $\sqrt{2 f}$,

$$
\left|\frac{d \boldsymbol{Q}}{d t^{\prime}}\right|=\left|\frac{d \boldsymbol{Q}}{d t} \frac{d t}{d t^{\prime}}\right|=\sqrt{2 f},
$$

whence,

$$
\frac{v}{\sqrt{f}} \frac{d t}{d t^{\prime}}=\sqrt{2 f}, \quad \text { i.e., } \quad \frac{d t^{\prime}}{d t}=\frac{v}{\sqrt{2} f} .
$$

Then $\boldsymbol{Q}=\boldsymbol{Q}\left(t^{\prime}\right)$, the lifted motion with respect to this new time variable, is indeed a Newtonian motion, that is, the particles positions $\overrightarrow{\mathrm{Q}}_{\alpha}, \alpha=1, \ldots, N$, forming the configuration $\boldsymbol{Q}$ satisfy Newton's equations

$$
m_{\alpha} \frac{d^{2} \overrightarrow{\mathrm{Q}}_{\alpha}}{d t^{\prime 2}}=-\vec{\nabla}_{\alpha} V\left(\overrightarrow{\mathrm{Q}}_{1}, \ldots \overrightarrow{\mathrm{Q}}_{N}\right) .
$$

One may wonder about the status of the time change (32). If one considers time to be absolute, $\boldsymbol{Q}=\boldsymbol{Q}(t)$ and $\boldsymbol{Q}^{\prime}=\boldsymbol{Q}^{\prime}(t)$ are two different motions. But if one takes a relational view about time, analogous to the relational view about space that we started with, $\boldsymbol{Q}=\boldsymbol{Q}(t)$ and $\boldsymbol{Q}^{\prime}=\boldsymbol{Q}^{\prime}(t)$ are the same motion. In other words, if time is relational, changes of speed, such as that given by 32 , provide equivalent representations of the same motion. Accordingly, the use of one time variable instead of another is a matter of convenience, analogous to the choice of a gauge. The choice of time variable for which Newton's equations (33) hold is the gauge fixing condition that leads from the invariant gauge to the Newton gauge; for the sake of simplicity, from now on we shall call it $t$ instead of $t^{\prime}$.

The invariant gauge has been defined by requiring that the path be horizontal. It turns out that this is equivalent to the following conditions:

$$
\begin{aligned}
& \sum_{\alpha=1}^{N} m_{\alpha} d \overrightarrow{\mathrm{Q}}_{\alpha}=0 \\
& \sum_{\alpha=1}^{N} m_{\alpha} \overrightarrow{\mathrm{Q}}_{\alpha} \times d \overrightarrow{\mathrm{Q}}_{\alpha}=0 \\
& \sum_{\alpha=1}^{N} m_{\alpha} \overrightarrow{\mathrm{Q}}_{\alpha} \cdot d \overrightarrow{\mathrm{Q}}_{\alpha}=0 .
\end{aligned}
$$


To see how this comes about, let

$$
\delta \overrightarrow{\mathrm{Q}}_{\alpha}=\vec{\epsilon}+\vec{\theta} \times \overrightarrow{\mathrm{Q}}_{\alpha}+\lambda \overrightarrow{\mathrm{Q}}_{\alpha}
$$

where $\vec{\epsilon}, \vec{\theta}$, and $\lambda$ are the infinitesimal parameters of a translation, a rotation and a dilation respectively, and let $\delta \boldsymbol{Q}=\left(\delta \overrightarrow{\mathbf{Q}}_{1} \ldots, \delta \overrightarrow{\mathbf{Q}}_{N}\right)$. Then

$$
\boldsymbol{Q} \rightarrow \boldsymbol{Q}+\delta \boldsymbol{Q}
$$

is an infinitesimal vertical transformation. Since the infinitesimal motion displacement $d \boldsymbol{Q}$ is purely horizontal, it must be orthogonal to $\delta \boldsymbol{Q}$, i.e., $g(d \boldsymbol{Q}, \delta \boldsymbol{Q})=0$, which implies that

$\vec{\epsilon} \cdot \sum_{\alpha=1}^{N} m_{\alpha} d \overrightarrow{\mathbf{Q}}_{\alpha}+\vec{\theta} \cdot \sum_{\alpha=1}^{N} m_{\alpha} \overrightarrow{\mathbf{Q}}_{\alpha} \times d \overrightarrow{\mathbf{Q}}_{\alpha}+\lambda \sum_{\alpha=1}^{N} m_{\alpha} \overrightarrow{\mathbf{Q}}_{\alpha} \cdot d \overrightarrow{\mathbf{Q}}_{\alpha}=0$.

This equality is satisfied (for all $\epsilon, \vec{\theta}$, and $\lambda$ ) only if the terms multiplying $\vec{\epsilon}, \vec{\theta}$, and $\lambda$ are separately zero, whence (34), (35), and (36).

The constraints (34), (35), and (36) have a natural meaning for a theory aimed at describing the universe as a whole. So to speak, they minimize the amount of motion when the universe is described in the invariant gauge.

Moreover, the constraints (34) and (36) are equivalent, respectively, to the requirements that the motion $\mathbf{Q}(t)$ is such that the center of mass $\left(\sum m_{\alpha}\right)^{-1} \sum m_{\alpha} \overrightarrow{\mathrm{Q}}_{\alpha}$ and the moment of inertia about the origin $\sum m_{\alpha} \overrightarrow{\mathrm{Q}}_{\alpha}^{2}$ don't change. Clearly, these are natural gauge fixing choices corresponding to translational and dilational (scaling) symmetry. However, there can be no function on absolute configuration space which corresponds in a similar way to (35). The constraint (35) does not correspond to the constancy of a function on absolute configuration space $5^{5}$

In the Newton gauge, (34), (35), and (36) can be expressed in terms of the familiar total momentum $\vec{P}$, total angular momentum $\vec{J}$ and (maybe less familiar) dilational momentum $D$ as

$$
\begin{aligned}
\overrightarrow{\mathrm{P}} & =\sum_{\alpha=1}^{N} m_{\alpha} \frac{d \overrightarrow{\mathrm{Q}}_{\alpha}}{d t}=0 \\
\overrightarrow{\mathrm{J}} & =\sum_{\alpha=1}^{N} m_{\alpha} \overrightarrow{\mathrm{Q}}_{\alpha} \times \frac{d \overrightarrow{\mathrm{Q}}_{\alpha}}{d t}=0 \\
D & =\sum_{\alpha=1}^{N} m_{\alpha} \overrightarrow{\mathrm{Q}}_{\alpha} \cdot \frac{d \overrightarrow{\mathrm{Q}}_{\alpha}}{d t}=0 .
\end{aligned}
$$

\footnotetext{
5 This corresponds to the fact that the subspaces of the tangent spaces (at the points in absolute configuration space) orthogonal to the fibers don't correspond to a foliation of absolute configuration space into submanifolds orthogonal to the fibers. This is related to the fact that the curvature of the connection relating the tangent spaces sitting at different points is non-vanishing and this, in its turn, is related to the Berry phase.
}

\section{Some Remarks on Relational Space and Relational Time}

The first simple moral to draw form the foregoing is that free motion on shape space, i.e., for interaction energy $\mathscr{V}=0$, leads to interaction energy $V \neq 0$ in the Newton gauge and so to an interacting particle dynamics in absolute spacetime (governed by Newton's laws (33)). In other words, the geometry on shape space defined by the conformal factor $f$ manifests itself as potential energy $V$ among the particles in the Newton gauge.

This remarkable fact is a direct consequence of the two main features of the theory under consideration. One is our starting point, namely that shape space is fundamental, that is, that space is relational. The other one has emerged in the analysis of how shape dynamics appears in the Newton gauge: motions following the same path with different speeds are indeed the same motion. And this corresponds to time being relational.

This remarkable fact notwithstanding, one may still wonder what sort of motion in absolute space corresponds to a shape dynamics with potential energy $\mathscr{V} \neq 0$. To answer to this question, let us go back to equations (18) or (19) defining interacting motion in shape space. Clearly, these equations are not in harmony with relational time: the acceleration in the LHS of (18) or the Euler-Lagrange equations arising from (19) rely on absolute time. On the other hand, the characterization of motion in terms of the Jacobi principle fits nicely with relational time. Adapted to the present case, this principle says that the path followed by a motion in shape space is the path that minimizes

$$
\int_{q_{1}}^{q_{2}} \sqrt{\mathscr{E}-\mathscr{V}}|d q|
$$

where $\mathscr{E}$ is any given fixed constant. And this is in complete harmony with relational time: if time is relational all that matters is the path and not the speed along the path. Note, however, that for the relational dynamics defined by 42 changing the potential by adding a constant changes the dynamics, unlike the dynamics defined by 18 or 19 .

Moreover, if interacting motion is defined according to 42), it will still be free motion, although with respect to a different metric: the one defined by the conformal factor $f_{\mathscr{E}, \mathscr{V}}=(\mathscr{E}-\mathscr{V}) f$ (with $\mathscr{V}(\boldsymbol{q})=\mathscr{V}(q)$, for any point $\boldsymbol{q}$ on the fiber above $q$ ). As for the starting question concerning how the motion appears in the Newton gauge, the answer is rather obvious: just as above, but now for the conformal factor $f_{\mathscr{E}, \mathscr{V}}=(\mathscr{E}-\mathscr{V}) f$.

The motion on shape space characterized by $(42)$ is defined for any potential $\mathscr{V}$ on shape space; in particular, it is defined for $\mathscr{V}+\mathscr{E}$. So the constant $\mathscr{E}$ can be absorbed in the potential; that is, without any loss of generality, we may set $\mathscr{E}=0$ and consider only $f_{\mathscr{V}}=-\mathscr{V} f$. In this regard, it is important to observe that changing the potential by a constant changes the conformal factor and thus 
changes the dynamics. This is a peculiar aspect of relational mechanics (relational space and relational time), as opposed to the usual Newtonian mechanics (absolute space and absolute time), where a change of the potential by a constant does not change Newton's laws.

\section{Newtonian Gravitation}

In the previous sections we found that in the Newton gauge, when the physical law on shape space is free motion (or even non-free motion), the potential $V=-f$ appears, where $f$ is the conformal factor. We mentioned some choices for $f$ in Sect IID. No such choices, which are necessarily functions homogenous of degree -2 , seem to yield exactly the Newtonian gravitational potential $U_{g}$. While we believe the detailed exploration of the implications of the models discussed here is worthwhile, we nonetheless regard the models explored in this paper, both classical and quantum, as toy models, so that such an analysis of them, with the expectation of recovering well established physics, might be somewhat inappropriate or premature.

However, it should be observed that some of the conformal factors given in Sect. IID, e.g., $f_{a}$ and $f_{g}$, indeed give rise to a force law in the Newton gauge that is very close to that of the Newtonian gravitational potential. Note for example that for the conformal factor $f_{g}$ the corresponding potential is of the form $V_{g}=\mathrm{L}^{-1} U_{g}$, where $U_{g}$ is the Newton gravitational potential and $\mathrm{L}$ in the Newton gauge is a constant of the motion. The force arising from this potential adds to the Newtonian force a very small centripetal correction that allows $I=\mathrm{L}^{2}$, the moment of inertia about the center of mass, to remain constant 4].

\section{E. Gauge Freedom, Symmetry Breaking, and Newton's Bucket}

The structures in an absolute space involved in the formulation of the geometry of shape space - in particular, the metric $g$ given by the conformal factor - are invariant under translations, rotations, and scaling. So, of course, is the classical dynamics on shape space, since, by construction, translations, rotations, and scaling act trivially on shape space. The procedure defining the invariant gauge (Sect. VB respects all of these symmetries. But scale invariance is broken in the Newton gauge because the time change 32 involved in the transition from the invariant gauge to the Newton gauge depends on the scale via $f$. This illustrates the obvious fact that the symmetries of the law of motion arising from the fundamental dynamics on shape space by a choice of gauge depends on the particular details defining that gauge.

A much larger class of symmetries for the shape space dynamics - also acting trivially — involves an independent group action $\mathrm{g} \in G$ at each "time" (but not so indepen- dent that smoothness is lost). The most important and familiar of these symmetries, when applied in a particular gauge, are uniformly growing translations (corresponding to Galilean boosts) and uniformly growing rotations (corresponding to the use of a rotating coordinate system or frame of reference). The former are a symmetry of the law of motion of the Newton gauge (ignoring the constraints (39)-(41), which are obviously not preserved under boosts), since a change in position that depends linearly on time produces no change in the acceleration. The latter, however, is not a symmetry of the Newtonian law of motion.

The behavior of Newton's bucket, which has been used to argue against a relational understanding of space, is thus seen, in fact, to be a natural consequence of the relational view. That behavior is a consequence of Newtonian-like laws akin to those that emerge as the description in the Newton gauge of the fundamental dynamics on shape space. However, in the Newton gauge the total angular momentum of the universe must vanish, and this is incompatible with a (non-negligible) uniform rotation of the "fixed stars." In a gauge corresponding to applying a uniformly growing rotation to the motion of the Newton gauge, the Newtonian law of motion is not obeyed, though the motion so obtained remains entirely compatible with the fundamental dynamics on shape space, a dynamics for which the behavior of the bucket depends essentially on its motion relative to that of the fixed stars.

\section{THE EMERGENCE OF ABSOLUTE SPACE AND TIME IN THE QUANTUM CASE}

\section{A. Gauge Freedom in the Quantum Case}

As in the classical case, also the quantum theory is about shapes, if one takes the standpoint of Bohmian mechanics. In this formulation of quantum mechanics, the role of the wave function is that of governing the motion of shapes. Moreover, as in the classical case, there is gauge freedom: a huge host of motions in absolute space $\mathcal{Q}$ are compatible with Bohmian motion in shape space 2. But now the presence of the wave function makes the freedom larger and subtler at the same time, as we shall explain in the following.

\section{B. The Schrödinger Gauge}

Let $Q=Q(t)$ be a Bohmian motion in shape space, that is, a solution of 25 with the wave function $\Psi$ being a solution of Schrödinger's equation 26 on shape space. For simplicity, we shall assume that $\mathscr{V}=0$ so that $(26)$ becomes

$$
i \hbar \frac{\partial \psi}{\partial t}=\mathrm{H} \psi, \quad \mathrm{H}=-\frac{\hbar^{2}}{2} \Delta_{B}
$$


with $\Delta_{B}$ the Laplace-Beltrami operator on shape space.

As in the classical case, we wish to characterize motions in absolute space that are compatible with motions in shape space, that is, motions $\boldsymbol{Q}=\boldsymbol{Q}(t)$ in $\mathcal{Q}$ that project down to $Q=Q(t)$ in 2 , i.e., such that

$$
\pi(\boldsymbol{Q}(t))=Q(t)
$$

where $\pi$ is the canonical projection from $\mathcal{Q}$ space to $\mathcal{Q}$. Clearly, there are a great many possibilities for compatible motions in absolute configuration space.

As in the classical case, one may restrict the possibilities by considering natural gauges. And as in the classical case where one looks for gauges such that the absolute motions satisfy Newton's equations, in the quantum case we now look for gauges such that the compatible motions on $\mathcal{Q}$ are themselves Bohmian motions, i.e. motions generated by a wave function in the usual sort of way.

For example, suppose that we proceed as in the classical case and take a horizontal lift of a motion $Q=Q(t)$ in shape space, that is, an absolute motion for which the infinitesimal displacements $d \boldsymbol{Q}$ are all horizontal. Let us now consider the lift to $\mathcal{Q}$ of a wave function $\Psi$ on $\mathcal{Q}$, namely, the wave function $\widehat{\Psi}_{1}$ on absolute configuration space such that

$$
\widehat{\Psi}_{1}(\boldsymbol{q})=\Psi(q)
$$

for any point $\boldsymbol{q}$ on the fiber above $q$. Let $\nabla_{g}$ be the gradient with respect to the invariant measure (4). Then the vector $\nabla_{g} \widehat{\Psi}_{1}(\boldsymbol{q})$ in $\mathcal{Q}$ is horizontal and the motions on $\mathcal{Q}$ defined by

$$
\frac{d \boldsymbol{Q}}{d t}=\hbar \operatorname{Im} \frac{\nabla_{g} \widehat{\Psi}_{1}}{\widehat{\Psi}_{1}}
$$

are horizontal lifts of motions on 2 . So, in the quantum case, horizontality is immediate.

Let us now consider the time evolution of the lifted wave function $\widehat{\Psi}_{1}$ on 2 . Let $\widehat{\Delta}_{B}$ be a lift to absolute configuration space of the Laplace-Beltrami operator $\Delta_{B}$ on shape space, namely an operator on $\mathcal{Q}$ such that

$$
\widehat{\Delta}_{B} \widehat{\Psi}_{1}=\Delta_{B} \Psi
$$

Then

$$
i \hbar \frac{\partial \widehat{\Psi}_{1}}{\partial t}=\widehat{\mathrm{H}}_{1} \widehat{\Psi}_{1}, \quad \text { with } \quad \widehat{\mathrm{H}}_{1}=-\frac{\hbar^{2}}{2} \widehat{\Delta}_{B} .
$$

It might seem natural to guess that $\widehat{\Delta}_{B}$ coincides with $\Delta_{g}$, the Laplace-Beltrami operator with respect to $g$, but this is wrong; nor is $\widehat{H}_{1}$ a familiar sort of Schrödinger Hamiltonian, with or without a potential term.

While $\widehat{\Psi}_{1}$ need not obey any familiar Schrödingertype equation, one may ask whether there exists a gauge equivalent wave function that does. By gauge equivalent wave function we mean this: If one writes $\widehat{\Psi}_{1}$ as $R e^{(i / \hbar) S}$ one sees that the velocity field given by 46 is just $\nabla_{g} S$, so transformations of the wave function

$$
\widehat{\Psi}_{1} \rightarrow \widehat{\Psi}_{1}^{\prime}=F \widehat{\Psi}_{1}
$$

where $F$ is a positive function, do not change its phase and thus the velocity.

It turns out that there exists a positive function $F$ such that $\widehat{\Psi}_{3}=F \widehat{\Psi}_{1}$ (why we use 3 here instead of 2 will be clearer in Sect. VIC) satisfies a Schrödinger type equation on absolute configuration space for a suitable potential $V$, namely,

$$
i \hbar \frac{\partial \widehat{\Psi}_{3}}{\partial t}=\widehat{\mathrm{H}}_{3} \widehat{\Psi}_{3}
$$

with

$$
\begin{aligned}
\widehat{\mathrm{H}}_{3} & =-\frac{\hbar^{2}}{2} \sum_{\alpha=1}^{N} \vec{\nabla}_{\alpha} \cdot \frac{1}{f m_{\alpha}} \vec{\nabla}_{\alpha}+V \\
& =-\frac{\hbar^{2}}{2} \nabla \cdot \frac{1}{f} \nabla+V,
\end{aligned}
$$

where $\boldsymbol{\nabla}$ and $\boldsymbol{\nabla} \cdot$ are the gradient and divergence with respect to the mass-weighted Eucidean metric (2), i.e.,

$$
\boldsymbol{\nabla}=\left(\frac{1}{m_{1}} \vec{\nabla}_{1}, \ldots, \frac{1}{m_{N}} \vec{\nabla}_{N}\right)
$$

and

$$
\nabla \cdot=\left(\vec{\nabla}_{1}, \ldots, \vec{\nabla}_{N}\right) \cdot
$$

Here $f$ is the conformal factor, and

$$
\begin{aligned}
V & =V_{1}+V_{2} \\
V_{1} & =-\frac{\hbar^{2}}{2} \frac{\widehat{\Delta}_{B} J^{1 / 2}}{J^{1 / 2}} \\
V_{2} & =-\frac{\hbar^{2}}{2} f^{\frac{n}{4}} \Delta_{g}\left(f^{-\frac{n}{4}}\right),
\end{aligned}
$$

with

$$
J=\mathrm{L} f^{7 / 2} \sqrt{\operatorname{det} \mathrm{M}},
$$

where $\mathrm{L}=\mathrm{L}(\boldsymbol{q})$ is given by equation $(9)$ and $\mathrm{M}=\mathrm{M}(\boldsymbol{q})$ is the tensor of inertia of the configuration $\boldsymbol{q}$ about any orthogonal cartesian system $x, y, z$ with origin in its center of mass and with matrix elements given by 12 , and where $\widehat{\Delta}_{B}$ is the canonical lift 65 .

We shall now describe what we think is appropriate to be called the Schrödinger gauge, the true quantum analogue of the Newton gauge. If we take into account that time is relational, as we should, the fundamental equation for the wave function on shape space is presumably the stationary equation

$$
-\frac{\hbar^{2}}{2} \Delta_{B} \Psi=\mathscr{E} \Psi
$$


where, for simplicity, we have set $\mathscr{V}=0$ and $\mathscr{E}$ is any given fixed constant (for example $\mathscr{E}=0$ ).

As before, let $\widehat{\Psi}_{1}$ be the lift of $\Psi$ to $\mathcal{Q}$, so that $\widehat{\Psi}_{1}$ satisfies the equation

$$
\left(\widehat{\mathrm{H}}_{1}-\mathscr{E}\right) \widehat{\Psi}_{1}=0
$$

with $\widehat{\mathrm{H}}_{1}$ a lift of $\mathrm{H}$ as in 48 , and the evolution on the absolute configuration $\mathcal{Q}$ is still given by (46). But now, for relational time, motions following the same path with different speeds are the same motion. So, in the formula for the gradient on the right hand side of $46, \nabla_{g}=$ $f^{-1} \nabla$, we may regard $f$ as a change of speed defining a new time variable that for the sake of simplicity we shall still call $t$ ("random time change"). Then in absolute space the guiding equation (46) becomes

$$
\frac{d \vec{Q}_{\alpha}}{d t}=\frac{\hbar}{m_{\alpha}} \operatorname{Im} \frac{\vec{\nabla}_{\alpha} \widehat{\Psi}_{1}}{\widehat{\Psi}_{1}} .
$$

Again, $\widehat{\Psi}_{1}$ need not obey any familiar stationary Schrödinger-type equation. However, as before, we may exploit gauge freedom to transform 60 into a stationary Schrödinger-type equation. Indeed, we have an even greater gauge freedom in changing the wave function and the Hamiltonian, as will be shown below. In particular, there is a gauge, the Schrödinger gauge, in which 59 becomes

$$
\widehat{\mathrm{H}}_{S} \Phi=0
$$

with

$$
\widehat{\mathrm{H}}_{S}=-\frac{\hbar^{2}}{2} \nabla^{2}+U
$$

where $\nabla^{2}=\boldsymbol{\nabla} \cdot \boldsymbol{\nabla}$ is the mass-weighed Euclidean Laplacian, and

$$
U=f\left(V_{1}-\mathscr{E}\right)-\frac{\hbar^{2}}{8} \frac{n-2}{n-1} f R_{g},
$$

where $R_{g}$ is the scalar curvature of the invariant metric $g$.

\section{Proofs of the Transitions to the Different Hamiltonians}

We shall now provide proofs of the transitions from Hamiltonian $\widehat{\mathrm{H}}_{1}$ in equations (48) and (60) to Hamiltonian $\widehat{\mathrm{H}}_{3}$, given by $(52)$, in equation $(50)$, and Hamiltonian $\widehat{\mathrm{H}}_{S}$, given by 63 , in equation 62 . The material presented here and in the following subsections is of a more mathematical character and could be skipped in first reading.

The lift to $\mathcal{Q}$ of the Laplace-Beltrami operator $\Delta_{B}$ on $\mathcal{Q}$ is by no means unique. There is however a "canonical lift" given by the formula

$$
\widehat{\Delta}_{B}=\mathfrak{J} \operatorname{div}_{g} \mathfrak{J}^{-1} \operatorname{grad}_{g},
$$

where $\mathfrak{J}=\mathfrak{J}(\boldsymbol{q})$ is a positive function on $\mathcal{Q}$ (unique up to a constant multiple), $\operatorname{grad}_{g}$ is the gradient, given by (using the Einstein summation convention)

$$
\left(\operatorname{grad}_{g}\right)^{i}=g^{i j} \partial_{j}
$$

in a coordinate basis $\left(\partial_{1}, \ldots, \partial_{n}\right)$, and $\operatorname{div}_{g}$ is the divergence whose action on a vector field $\mathbf{Y}=\left(Y^{1}, \ldots, Y^{n}\right)$ in the coordinate basis $\left(\partial_{1}, \ldots, \partial_{n}\right)$ is

$$
\operatorname{div}_{g} \mathbf{Y}=\frac{1}{\sqrt{|g|}} \partial_{i} \sqrt{|g|} Y^{i}
$$

where $|g|=\left|\operatorname{det}\left(g_{i j}\right)\right|$ is the absolute value of the determinant of the metric tensor $g_{i j}$ in the given local coordinates. The existence of a positive $\mathfrak{J}$ such that 65 defines a lift of $\Delta_{B}$ will be proven in Sect. VIE and formula (58) for $\mathfrak{J}$ will be derived in Sect VIF. We call $\mathfrak{J}$ the shape Jacobian.

While $\widehat{\Delta}_{B}$ does not coincide with $\Delta_{g}$, the LaplaceBeltrami operator on $\mathcal{Q}$, it is a minimal modification thereof. Just compare 65 with $\Delta_{g}$ as "div-grad" operator, i.e., in local coordinates,

$$
\begin{aligned}
\Delta_{g}=\operatorname{div}_{g} \operatorname{grad}_{g} & =\frac{1}{\sqrt{|g|}} \partial_{i} \sqrt{|g|} g^{i j} \partial_{j} \\
& =\frac{1}{f^{n / 2}} \nabla \cdot f^{(n / 2)-1} \nabla,
\end{aligned}
$$

where in the second equality we have made explicit the invariant metric $g=f g_{e}$, with $g_{e}$ given by (2), in Euclidean particle coordinates: $g_{i j}=f m_{\alpha_{i}} \delta_{i j}$, so that $\sqrt{|g|}=\left(m_{1} \cdots m_{N}\right)^{3 / 2} f^{n / 2}$, with $n=3 N$, and $g^{i j}=f^{-1} m_{\alpha_{i}}^{-1} \delta_{i j}$. Similarly,

$$
\begin{aligned}
\widehat{\Delta}_{B} & =\frac{\mathfrak{J}}{\sqrt{|g|}} \partial_{i} \frac{\sqrt{|g|}}{\mathfrak{J}} g^{i j} \partial_{j} \\
& =\frac{\mathfrak{J}}{f^{n / 2}} \nabla \cdot \frac{f^{(n / 2)-1}}{\mathfrak{J}} \nabla
\end{aligned}
$$

Note that $\Delta_{g}$ is self-adjoint on $L^{2}\left(d \mu_{g}\right)$, the set of functions on $\mathcal{2}$ square integrable with respect to the volume element defined by the metric $g$,

$$
d \mu_{g}=\sqrt{|g|} d x_{1} \cdots d x_{n} \propto f^{n / 2} d^{3} \overrightarrow{\mathbf{q}}_{1} \cdots d^{3} \overrightarrow{\mathbf{q}}_{N}
$$

$(n=3 N)$. In contrast, $\widehat{\Delta}_{B}$ is self adjoint with respect to the volume element $d \mu=\mathfrak{J}^{-1} d \mu_{g}$.

Let us now consider the effect of the gauge transformation 49 on $\widehat{\mathrm{H}}_{1}=-\left(\hbar^{2} / 2\right) \widehat{\Delta}_{B}$. Since $\widehat{\Psi}_{1} \rightarrow \widehat{\Psi}_{2}=F \widehat{\Psi}_{1}$ is a unitary transformation

$$
U: L^{2}(d \mu) \rightarrow L^{2}\left(F^{-2} d \mu\right)
$$

the effect of 49 is to transform $\widehat{\mathrm{H}}_{1}$ into the unitarily equivalent operator

$$
\widehat{\mathrm{H}}_{2}=U \widehat{\mathrm{H}}_{1} U^{-1}=F \widehat{\mathrm{H}}_{1} F^{-1},
$$


so that $\left\{\widehat{\mathrm{H}}_{1}, \widehat{\Psi}_{1}\right\}$ and $\left\{\widehat{\mathrm{H}}_{2}, \widehat{\Psi}_{2}\right\}$ provide equivalent description of the dynamics.

A natural question is whether there is an equivalent description such that $\widehat{\mathrm{H}}_{2}$ is Schrödinger-like with some potential. The key to answering this question is the following theorem concerning second order partial differential operators (see the Appendix for a proof): Suppose $H_{1}$ and $H_{2}$ are second order partial differential operators, both self-adjoint with respect to the same measure. If they have the same pure 2nd derivative parts then

$$
H_{2}=H_{1}+V \text {. }
$$

Moreover, if $H_{1} 1=0$ (no constant part) then $V=H_{2} 1$.

We first apply this theorem to the operators $H_{1}=$ $-\left(\hbar^{2} / 2\right) \Delta_{g}$ and $H_{2}=\widehat{\mathrm{H}}_{2}$, unitarily equivalent to $\widehat{\mathrm{H}}_{1}$ according to (74). Choosing $F=\mathfrak{J}^{-1 / 2}$ in (74), $\mathrm{H}_{2}$ is self-adjoint with respect to $\mu_{g}$. (According to (73), this operator is self-adjoint with respect to $\mathfrak{J} d \mu=\mathfrak{J} \mathfrak{J}^{-1} d \mu_{g}=$ $\left.d \mu_{g}.\right)$ So, $H_{1}$ and $H_{2}$ so defined are self-adjoint with respect to the same measure, have the same pure 2 nd derivative parts (namely, $-\left(\hbar^{2} / 2\right) f^{-1} \boldsymbol{\nabla} \cdot \boldsymbol{\nabla}$ ) and $H_{1} 1=$ 0 . Thus, according to the theorem stated above,

$$
\widehat{\mathrm{H}}_{2}=-\frac{\hbar^{2}}{2} \Delta_{g}+V_{1}
$$

with

$$
V_{1}=-\frac{\hbar^{2}}{2} \frac{\widehat{\Delta}_{B} \mathfrak{J}^{1 / 2}}{\mathfrak{J}^{1 / 2}}
$$

Let us now perform a further transformation on $\widehat{\mathrm{H}}_{2}$ to make it unitarily equivalent to the operator $\widehat{H}_{3}$ (see 52 ), which is self-adjoint with respect to the Lebesgue measure $d^{3} \overrightarrow{\mathrm{q}}_{1} \cdots d^{3} \overrightarrow{\mathrm{q}}_{N}$. Observing the form $\sqrt{69}$ of $\Delta_{g}$, the desired transformation is

$$
\begin{aligned}
\widehat{\mathrm{H}}_{2} \rightarrow \widehat{\mathrm{H}}_{3} & =f^{n / 4} \widehat{\mathrm{H}}_{2} f^{-n / 4} \\
& =-\frac{\hbar^{2}}{2} f^{n / 4} \Delta_{g} f^{-n / 4}+V_{1} \\
& \equiv H_{2}+V_{1} .
\end{aligned}
$$

Consider now the operator

$$
H_{1}=-\frac{\hbar^{2}}{2} \nabla \cdot \frac{1}{f} \nabla
$$

and note that $H_{1}$ and $H_{2}$ have the same pure 2 nd derivative parts, are self-adjoint with respect to the same measure (the Lebesgue measure) and $H_{1} 1=0$. Thus,

$$
H_{2}=H_{1}+V_{2}
$$

with

$$
V_{2}=H_{2} 1=-\frac{\hbar^{2}}{2} f^{\frac{n}{4}} \Delta_{g}\left(f^{-\frac{n}{4}}\right)
$$

Finally, by inserting $H_{2}$ into 80, , we get

$$
\widehat{\mathrm{H}}_{3}=H_{1}+V_{2}+V_{1}=-\frac{\hbar^{2}}{2} \nabla \cdot \frac{1}{f} \nabla+V_{1}+V_{2},
$$

which is formula (52) with $V_{1}$ and $V_{2}$ given by (56) and (57).

Consider now the stationary equation corresponding to equation (76), namely

$$
\left(\widehat{\mathrm{H}}_{2}-\mathscr{E}\right) \widehat{\Psi}_{2}=0
$$

and observe that now we may allow a broader class of transformations $\left\{\widehat{\mathrm{H}}_{2}, \widehat{\Psi}_{2}\right\} \rightarrow\left\{\widehat{\mathrm{H}}_{S}, \Phi\right\}$ leading to an equivalent description of the dynamics. More precisely, with a change of $\widehat{\Psi}_{2}$ according to 49 ,

$$
\widehat{\Psi}_{2} \rightarrow \widehat{\Psi}_{S}=F \widehat{\Psi}_{2}
$$

with $F>0$, we need not demand now that the Hamiltonian gets transformed according to (74); the more general change

$$
\left(\widehat{\mathrm{H}}_{2}-\mathscr{E}\right) \rightarrow \widehat{\mathrm{H}}_{S}=G\left(\widehat{\mathrm{H}}_{2}-\mathscr{E}\right) F^{-1}
$$

with $G>0$ not necessarily equal to $F^{-1}$ suffices. Recalling (76), we have

$$
\widehat{\mathrm{H}}_{S}=H_{2}+G F^{-1}\left(V_{1}-\mathscr{E}\right),
$$

with now $\mathrm{H}_{2}$ defined as

$$
H_{2}=-\frac{\hbar^{2}}{2} G \Delta_{g} F^{-1}
$$

Observing the form 69 of $\Delta_{g}$, for the choice

$$
F=f^{\frac{n-2}{4}}, \quad G=f^{\frac{n}{2}} f^{-\frac{n-2}{4}}=f^{\frac{n+2}{4}},
$$

$H_{2}$ has the same pure 2 nd derivative part as $H_{1} \equiv$ $-\left(\hbar^{2} / 2\right) \Delta$; moreover, $H_{1}$ and $H_{2}$ so defined are selfadjoint with respect to Lebesgue measure and $H_{1} 1=0$. Thus,

$$
H_{2}=-\frac{\hbar^{2}}{2} \nabla^{2}+V_{3}
$$

with

$$
V_{3}=-\frac{\hbar^{2}}{2} f^{\frac{n+2}{4}} \Delta_{g} f^{-\frac{n-2}{4}}
$$

The potential $V_{3}$ has a natural geometrical meaning. To see this, note that the scalar curvatures $R_{g}$ and $R_{\tilde{g}}$ of two conformally related metrics $g$ and $\tilde{g}=\Lambda g$ are related by the formula (see, e.g., [19])

$$
R_{\tilde{g}}=\Lambda^{-1}\left(R_{g}-\frac{4(n-1)}{n-2} \Lambda^{-\frac{n-2}{4}} \Delta_{g} \Lambda^{\frac{n-2}{4}}\right) .
$$


Letting $g$ be the invariant metric on $\mathcal{Q}, \tilde{g}$ be the Euclidean metric on 2 (so that $R_{\tilde{g}}=0$ ) and $\Lambda=f^{-1}$, we obtain that

$$
R_{g}=\frac{4(n-1)}{n-2} f^{\frac{n-2}{4}} \Delta_{g} f^{-\frac{n-2}{4}},
$$

whence

$$
V_{3}=-\frac{\hbar^{2}}{2} \frac{n-2}{4(n-1)} f R_{g} .
$$

Since $G F^{-1}=f$, we conclude that

$$
\widehat{\mathrm{H}}_{S}=-\frac{\hbar^{2}}{2} \nabla^{2}+f\left(V_{1}-\mathscr{E}\right)-\frac{\hbar^{2}}{8} \frac{n-2}{n-1} f R_{g},
$$

which coincides with 63 for $U$ given by (64). This completes the proofs of the transitions to the different Hamiltonians.

Note that while $V_{2}$ and $V_{3}$ do not depend on the shape Jacobian $\mathfrak{J}, V_{1}$ does. So, to find $V_{1}$, we have first to find an explicit formula for $\mathfrak{J}$. This will be done in Sect. VIE and Sect. VIF.

\section{Remarks on the Bohmian Motion in the Various Gauges}

We have already stated that the usual Bohmian motion associated with $\widehat{\mathrm{H}}_{2}$ is given by (46), see 23 . This is true also for $\widehat{\mathrm{H}}_{1}$ and $\widehat{\mathrm{H}}_{3}$. To see this, consider a Hamiltonian of the form

$$
H=-\frac{h^{2}}{2} F \nabla \cdot G \nabla+U,
$$

with $U$ a potential (multiplication operator), where $\nabla$ and $\nabla \cdot$ are, respectively, the gradient and the divergence with respect to a metric with associated volume element $d \mu$. Then $H$ is self-adjoint with respect to $F^{-1} d \mu$ and the velocity field generated by a solution $\widehat{\Psi}$ of the Schrödinger equation associated with $H$ is

$$
v=\hbar \operatorname{Im} \frac{F G \nabla \widehat{\Psi}}{\widehat{\Psi}} .
$$

Thus, in the "1-gauge" for $\widehat{\mathrm{H}}_{1}$ involving $\widehat{\Delta}_{B}$ given by (65), the Bohmian velocity (95) is indeed (46) (with $\widehat{\Psi}=\Psi_{1}$ ), since in this gauge $\nabla=\operatorname{grad}_{g}, \nabla \cdot=\operatorname{div}_{g}, F=\mathfrak{J}$ and $G=\mathfrak{J}^{-1}$. The same velocity arises in the "2-gauge" 76 . (with $\widehat{\Psi}=\widehat{\Psi}_{2}$ ), since now $F=G=1$ (and $\nabla=\operatorname{grad}_{g}$, $\nabla \cdot=\operatorname{div}_{g}$, as before), as well as in the "3-gauge" (84) (with $\widehat{\Psi}=\widehat{\Psi}_{3}$ ), since now $\nabla=\nabla, \nabla \cdot=\nabla \cdot$, the usual divergence, and $F=1, G=1 / f$, so that (95) equals (46) since $\nabla_{g}=f^{-1} \nabla$. On the other hand, in the "S -gauge" for $\widehat{\mathrm{H}}_{S}$ (the Schrödinger gauge) the Bohmian velocity is given by the usual formula (61), which, as already stated, arises from (46) after a time change.
We shall address the status of probability measures in Bohmian mechanics on shape space and in the various gauges in Sect. VIII. Here we shall just state some mathematical facts about the quantum equilibrium measures (Born's rule) associated with the Bohmian motions in the various gauges. These measures are the same in all the first three gauges, though they assume different forms. In each gauge, they are the quantum equilibrium measure associated with the solutions of the wave equation in that gauge.

By construction, and more explicitly, since 94 is selfadjoint with respect to $F^{-1} d \mu, \widehat{\mathrm{H}}_{1}, \widehat{\mathrm{H}}_{2}$, and $\widehat{\mathrm{H}}_{3}$ are selfadjoint with respect to the measures $\mathfrak{J}^{-1} d \mu_{g}, d \mu_{g}$, and $d \boldsymbol{q}$ (the Lebesgue measure on $\mathbf{2}$ ), respectively. Thus, the corresponding quantum equilibrium measures are, respectively,

$$
\left.\begin{array}{l}
\left|\widehat{\Psi}_{1}\right|^{2} \mathfrak{J}^{-1} d \mu_{g} \\
\left|\widehat{\Psi}_{2}\right|^{2} d \mu_{g} \\
\left|\widehat{\Psi}_{3}\right|^{2} d \boldsymbol{q}
\end{array}\right\} \equiv d \mu^{\widehat{\Psi}}
$$

The equality of these measures (up to a constant multiple) readily follows from the relations between the various gauges,

$$
\begin{aligned}
\widehat{\Psi}_{1}(\boldsymbol{q}) & =\Psi(q), \\
\widehat{\Psi}_{2} & =\mathfrak{J}^{-1 / 2} \widehat{\Psi}_{1}, \\
\widehat{\Psi}_{3} & =f^{n / 4} \widehat{\Psi}_{2},
\end{aligned}
$$

and formula 72 for $d \mu_{g}$. We finally note that in the Schrödinger gauge, as for the velocity, Born's probability law turns out to be the familiar one, namely,

$$
d \mu^{\widehat{\Psi}_{S}}=\left|\widehat{\Psi}_{S}\right|^{2} d \boldsymbol{q}
$$

Note that in going from the 3 -gauge to the $S$-gauge there is no change of measure for self-adjointness of the Hamiltonian, so that the change $\widehat{\Psi}_{3} \rightarrow \widehat{\Psi}_{S}$ leads in this case to a change in measure, $\mu^{\widehat{\Psi_{S}}} \neq \mu^{\widehat{\Psi}}$.

\section{E. Derivation of the Shape Jacobian}

We shall now derive formula 65. In order to do this, we shall compare the Laplace-Beltrami operator $\Delta_{g}$ on absolute configuration space $\mathbf{Q}$ with the LaplaceBeltrami operator $\Delta_{B}$ on shape space $2=2 / G$. This comparison would be easy if we could represent $\Delta_{g}$ in terms of coordinates $x^{i}=\left\{x^{H}, x^{V}\right\}$ such that the $x_{V}$ coordinate lines are all inside the $G$-fibers and the $x_{H}$ coordinate lines are orthogonal to them and thus yield a horizontal foliation. However, a coordinate system of this kind does not exist, not even locally, since the existence of a horizontal foliation of $\mathbf{Q}$ is precluded by the curvature of the horizontal connection arising from the 
rotations (see footnote 5). We shall not elaborate further on this. Nonetheless a splitting into horizontal and vertical components can be obtained by expressing the Laplace-Beltrami operator in terms of a basis formed by a suitable set of horizontal and vertical vector fields, as will be explained below ${ }^{6}$

First, we express gradient and divergence on a Riemannian manifold in terms of a general basis $\mathbf{X}$ of vector fields $\mathbf{X}_{i}, i=1, \ldots, n$. We replace (66) by

$$
\left(\operatorname{grad}_{g}\right)^{i}=g^{i j} \mathbf{X}_{j}
$$

where $g^{i j}=\left[g_{i j}\right]^{-1}$ with $g_{i j}=g\left(\mathbf{X}_{i}, \mathbf{X}_{j}\right)$, and replace 67$)$ with

$$
\operatorname{div}_{g} \mathbf{Y}=\left(\frac{1}{\sqrt{|g|}} \mathbf{X}_{i} \sqrt{|g|}+\omega^{k}\left(\left[\mathbf{X}_{k}, \mathbf{X}_{i}\right]\right)\right) Y^{i},
$$

where $|g|=\left|\operatorname{det}\left(g_{i j}\right)\right|,\left\{\boldsymbol{\omega}^{k}\right\}$ is the dual basis in the cotangent space, i.e., $\boldsymbol{\omega}^{k}\left(\mathbf{X}_{i}\right)=\delta_{i}^{k},[\cdot, \cdot]$ is the Lie bracket (or commutator) of vector fields, and $Y^{i}$ are the components of $\mathbf{Y}$ with respect to the basis $\mathbf{X}_{i} 7$ Accordingly, the Laplace-Beltrami operator 68 becomes

$$
\Delta_{g}=\left(\frac{1}{\sqrt{|g|}} \mathbf{X}_{i} \sqrt{|g|}+\omega^{k}\left(\left[\mathbf{X}_{k}, \mathbf{X}_{i}\right]\right)\right) g^{i j} \mathbf{X}_{j}
$$

which generalizes the standard formula 68 to an arbitrary basis.

Second, we specify a basis of vector fields that is adapted to the geometrical structure of absolute configuration space $\mathcal{Q}$ as a principal fiber bundle with base $\mathcal{Q}$ and fibers isomorphic to the similarity group $G$, in particular, to the orthogonal decomposition of the tangent space $T_{\boldsymbol{q}} \mathbf{Q}$ at any point $\boldsymbol{q}$ of $\mathbf{Q}$ into horizontal subspace $T_{q} \mathbf{Q}^{H}$ and vertical subspace $T_{q} \mathbf{Q}^{V}$ and the corresponding decomposition $T \mathbf{Q}=T \mathbf{Q}^{H} \oplus T \mathbf{Q}^{V}$ of the tangent bundle. In the horizontal subspace we choose as a basis the horizontal lift of a coordinate basis $X=\left\{X_{\alpha}\right\}$ in $2, \alpha=1, \ldots, n-7$. Note that while a lift of the vector field $X_{\alpha}$ is not unique (as any vector field on $\mathbf{Q}$ that projects down to $X_{\alpha}$ represents a lift of $X_{\alpha}$ ), there is only one horizontal lift of $X_{\alpha}$ which we shall denote by $\widehat{X}_{\alpha}$. These vector fields form the basis $\mathbf{X}_{H}=\left\{\widehat{X}_{\alpha}\right\} \equiv \widehat{X}$, $\alpha=1, \ldots, n-7$, in the horizontal subspace.

In the vertical subspace we choose a basis formed by vector fields that represent the action of the infinitesimal

\footnotetext{
${ }^{6}$ Moreover, the vertical vector fields that we shall need correspond to the Lie algebra of $G$, which is noncommutative, and thus do not arise from a coordinate system.

${ }^{7}$ Formula 102 is probably in the literature, but we have not succeeded in finding any reference. It is a straightforward consequence, for a manifold with a distinguished volume form (up to sign), of the fact that the divergence of a vector field times the volume form is the exterior derivative of the contraction of the vector field with the volume form.
}

generators of the group $G$ on $\mathbf{Q}$. More precisely, observe that the action $\boldsymbol{q} \rightarrow \mathrm{g}(\boldsymbol{q})$ of $G$ on $\boldsymbol{2}, \mathrm{g} \in G$, defines, for any given $\boldsymbol{q} \in \mathcal{Q}$, the map $\varphi_{\boldsymbol{q}}: G \rightarrow \mathcal{Q}$ given by $\varphi_{\boldsymbol{q}}(\mathrm{g})=$ $\mathrm{g}(\boldsymbol{q})$, and the differential $\varphi_{\boldsymbol{q}}^{\prime}$ of this map defines a map from $T_{e}(G)$, the tangent space to the identity $e$ of $G$, to $T_{q}$ 2. Since $T_{e}(G)$ is the Lie-algebra $\mathfrak{g}$ of the group $G$, the image under $\varphi_{q}^{\prime}$ of any element $L$ of $\mathfrak{g}$ is a tangent vector at $\boldsymbol{q}$ and, varying $\boldsymbol{q}$, one obtains the vector field $\overline{\mathrm{L}}$ on $\boldsymbol{Q}$ associated with $\mathrm{L}$. In particular, if $\mathrm{L}_{\beta}, \beta=1, \ldots 7$, are the generators of $\mathfrak{g}$, their images under $\varphi_{\boldsymbol{q}}^{\prime}$ form the basis $\mathbf{X}_{V}=\left\{\overline{\mathrm{L}}_{\beta}\right\}, \beta=1, \ldots 7$, in the vertical subspace. (It should be noted that the vertical vector fields so defined coincide with the image under $\varphi_{q}^{\prime}$ of the right invariant vector fields on $G$; in this regard, recall that the Liealgebra of the group can be equivalently defined as the Lie-algebra of the right —or left — invariant vector fields on $G$.)

Third, we rewrite the Laplace-Beltrami operator 103 in terms of the basis $\mathbf{X}=\left\{\mathbf{X}_{H}, \mathbf{X}_{V}\right\}$ using the compact (and slightly ambiguous) notation

$$
\begin{gathered}
\Delta_{g}=\frac{1}{\sqrt{|g|}} \mathbf{X}_{H} \sqrt{|g|} g^{H H} \mathbf{X}_{H}+\frac{1}{\sqrt{|g|}} \mathbf{X}_{V} \sqrt{|g|} g^{V V} \mathbf{X}_{V} \\
+\boldsymbol{\omega}^{A}\left(\left[\mathbf{X}_{A}, \mathbf{X}_{A}\right]\right) g^{A A} \mathbf{X},
\end{gathered}
$$

where repeated upper and lower indexes $H$, resp., $V$, stands for summation over all elements of $\left\{\mathbf{X}^{H}\right\}$, resp., $\left\{\mathbf{X}^{V}\right\}$. In the last term the summation is over $A=H, V$. Note, that no mixed contributions $H-V$ occur, since the vertical and horizontal vector fields are orthogonal and thus $g^{H V}=0$. Consider now $\Delta_{g} \widehat{\Psi}_{1}$, the action of $\Delta_{g}$ on an invariant function $\widehat{\Psi}_{1}(\boldsymbol{q})=\Psi(q)$. Since the second term in 104 is purely vertical, it gives no contribution. We rewrite the last term more explicitly, keeping only the non zero part of its action on invariant functions, to obtain

$$
\left(\boldsymbol{\omega}^{H}\left(\left[\mathbf{X}_{H}, \mathbf{X}_{H}\right]\right)+\boldsymbol{\omega}^{V}\left(\left[\mathbf{X}_{V}, \mathbf{X}_{H}\right]\right)\right) g^{H H} \mathbf{X}_{H} \widehat{\Psi}_{1} .
$$

The first term in the round brackets gives no contribution, in fact

$$
\left[\mathbf{X}_{H}, \mathbf{X}_{H}\right]=[\widehat{X}, \widehat{X}]=\widehat{[X, X]}+\text { Vertical }=\text { Vertical },
$$

where in the last equality we have used the fact that $X$ is a coordinate basis, and thus its elements commute; moreover, $\boldsymbol{\omega}^{H}$ (Vertical) is clearly zero. As for the second term, expressing the commutator of the vector fields by means of the Lie derivative $\mathcal{L}$,

$$
\left[\mathbf{X}_{V}, \mathbf{X}_{H}\right]=\mathcal{L}_{\mathbf{X}_{V}} \mathbf{X}_{H}=0
$$

by symmetry, i.e., the $G$-invariance of the vector fields $\mathbf{X}_{H}$. We conclude that for $\widehat{\Psi}_{1}$ an invariant function on 2, we obtain

$$
\Delta_{g} \widehat{\Psi}_{1}=\frac{1}{\sqrt{|g|}} \mathbf{X}_{H} \sqrt{|g|} g^{H H} \mathbf{X}_{H} \widehat{\Psi}_{1} .
$$


Fourth, we consider the Laplace-Beltrami operator on shape space acting on $\psi=\psi(q)$,

$$
\Delta_{B} \psi=\frac{1}{\sqrt{\left|g_{B}\right|}} X \sqrt{\left|g_{B}\right|} g^{B B} X \psi .
$$

Then the action of a lift of $\Delta_{B}$ on the invariant function $\widehat{\Psi}_{1}=\widehat{\Psi}_{1}(\boldsymbol{q})$ associated with $\psi$ is given by

$$
\widehat{\Delta}_{B} \widehat{\Psi}_{1}=\frac{1}{\sqrt{\left|g_{B}\right|}} \mathbf{X}_{H} \sqrt{\left|g_{B}\right|} g^{H H} \mathbf{X}_{H} \widehat{\Psi}_{1} .
$$

Comparing (109) with (107), we write this as

$$
\widehat{\Delta}_{B} \widehat{\Psi}_{1}=\mathfrak{J} \frac{1}{\sqrt{|g|}} \mathbf{X}_{H} \mathfrak{J}^{-1} \sqrt{|g|} g^{H}{ }^{H} \mathbf{X}_{H} \widehat{\Psi}_{1}
$$

with

$$
\mathfrak{J}=\frac{\sqrt{|g|}}{\sqrt{\left|g_{B}\right|}}
$$

Fifth (and finally), we consider the operator

$$
O=\mathfrak{J} \operatorname{div}_{g} \mathfrak{J}^{-1} \operatorname{grad}_{g},
$$

with $\mathfrak{J}$ given by (111), and observe that

$$
\begin{gathered}
\mathfrak{J} \operatorname{div}_{g} \mathfrak{J}^{-1} \mathbf{Y}=\mathfrak{J}\left(\frac{1}{\sqrt{|g|}} \mathbf{X}_{i} \sqrt{|g|}+\omega^{k}\left(\left[\mathbf{X}_{k}, \mathbf{X}_{i}\right]\right)\right) \mathfrak{J}^{-1} Y^{i} \\
=\left(\frac{\mathfrak{J}}{\sqrt{|g|}} \mathbf{X}_{i} \mathfrak{J}^{-1} \sqrt{|g|}+\boldsymbol{\omega}^{k}\left(\left[\mathbf{X}_{k}, \mathbf{X}_{i}\right]\right)\right) Y^{i}
\end{gathered}
$$

Thus $O \widehat{\Psi}_{1}$, with $\widehat{\Psi}_{1}$ an invariant function, coincides with the right hand side of 1110 ) (for the same reasons that led us from (104) to (107)). Therefore $O$, i.e. (65), is a lift of $\Delta_{B}$, with the shape Jacobian $\mathfrak{J}$ given explicitly by equation (111).

\section{F. Computation of the Shape Jacobian}

Our last task is to derive formula 58 from equation (111) for the shape Jacobian $\mathfrak{J}$.

First, we observe that in the basis $\left\{\mathbf{X}_{H}, \mathbf{X}_{V}\right\}$ the metric $g$ has the block diagonal decomposition (slightly abusing notation)

$$
g=\left(\begin{array}{cc}
g_{V} & 0 \\
0 & g_{H}
\end{array}\right)
$$

where $g_{H}$ can be identified with $g_{B}$ and $g_{V}$ is the restriction of $g$ to the vertical vector fields. Since $|g|=\left|g_{V}\right|\left|g_{H}\right|$ and $\left|g_{B}\right|=\left|g_{H}\right|$, it follows from 1111 that

$$
\mathfrak{J}=\frac{\sqrt{|g|}}{\sqrt{\left|g_{B}\right|}}=\frac{\sqrt{\left|g_{V}\right|\left|g_{H}\right|}}{\sqrt{\left|g_{B}\right|}}=\sqrt{\left|g_{V}\right|},
$$

so that $\mathfrak{J}$ turns out to be the invariant volume density in the vertical subspace with respect to fiber volume element corresponding to $\mathbf{X}_{V}$. Moreover, by (6) we have that

$$
\mathfrak{J}=f^{7 / 2} \mathfrak{J}_{e},
$$

where $\mathfrak{J}_{e}$ is the vertical volume density for the massweighted Euclidean metric (2) instead of the invariant metric $g$ involving the conformal factor $f$. For this we have

$$
\mathfrak{J}_{e}(\boldsymbol{q})=\operatorname{vol}\left(\mathbf{X}_{V}\right)(\boldsymbol{q}),
$$

the 7-dimensional (Euclidean) volume of the parallelepiped in $T_{q} 2^{V}$ generated by $\mathbf{X}_{V}$, i.e., by the vertical tangent vectors at $\boldsymbol{q}$ obtained by evaluating at $\boldsymbol{q}$ the 7 vector fields that generate $G$.

Second, we may split

$$
\mathbf{X}_{V}=\left(\mathbf{X}_{\mathrm{tr}}, \mathbf{X}_{\mathrm{rs}}\right)
$$

where $\mathbf{X}_{\text {tr }}$ refers to the 3 generators of translations and $\mathbf{X}_{\mathrm{rs}}$ to the 4 generators of rotations and scaling. However, the vector fields $\mathbf{X}_{\mathrm{rs}}$ are not in general orthogonal to those of $\mathbf{X}_{\mathrm{tr}}$. We therefore consider also $\widetilde{\mathbf{X}}_{\mathrm{rs}}=P_{\mathrm{tr}}^{\perp} \mathbf{X}_{\mathrm{rs}}$, the orthogonal projection of the vectors $\mathbf{X}_{\mathrm{rs}}$ into the orthogonal complement of the subspace of the tangent space corresponding to translations. We thus have that

$$
\begin{aligned}
\operatorname{vol}\left(\mathbf{X}_{V}\right) & =\operatorname{vol}\left(\mathbf{X}_{\mathrm{tr}}\right) \cdot \operatorname{vol}\left(\widetilde{\mathbf{X}}_{\mathrm{rs}}\right) \\
& \equiv \operatorname{vol}\left(\mathbf{X}_{\mathrm{tr}}\right) \widetilde{\mathfrak{J}}_{\mathrm{rs}}=\mathrm{const} \widetilde{\mathfrak{J}}_{\mathrm{rs}},
\end{aligned}
$$

since $\operatorname{vol}\left(\mathbf{X}_{\text {tr }}\right)$ is a constant, independent of $\boldsymbol{q}$ (which we may take to be 1 by letting the translation vectors in $\mathbf{X}_{\text {tr }}$ to be orthonormal). It should be observed that $\widetilde{\mathbf{X}}_{\mathrm{rs}}$ consists of the generators of rotations and scalings about the center of mass of the configuration $\boldsymbol{q}$. To see this, note that if we represent $\boldsymbol{q}$ in center of mass and relative coordinates $\widetilde{\boldsymbol{q}}=\boldsymbol{q}-\overrightarrow{\mathrm{q}}_{\mathrm{cm}}$, i.e., $\boldsymbol{q}=\left(\overrightarrow{\mathrm{q}}_{\mathrm{cm}}, \widetilde{\boldsymbol{q}}\right)$, then for any rotation or scaling $g$, we have that the action of $g$ on $\boldsymbol{q}$ is given in these coordinates by $\mathrm{g}\left(\overrightarrow{\mathrm{q}}_{\mathrm{cm}}, \widetilde{\boldsymbol{q}}\right)=\left(\mathrm{g}\left(\overrightarrow{\mathrm{q}}_{\mathrm{cm}}\right), \mathrm{g}(\widetilde{\boldsymbol{q}})\right)$, while for the corresponding action $\widetilde{\mathrm{g}}$ about the center of mass, $\widetilde{\mathrm{g}}\left(\overrightarrow{\mathrm{q}}_{\mathrm{cm}}, \widetilde{\boldsymbol{q}}\right)=\left(\overrightarrow{\mathrm{q}}_{\mathrm{cm}}, \mathrm{g}(\widetilde{\boldsymbol{q}})\right)$.

Third, we may split

$$
\widetilde{\mathbf{X}}_{\mathrm{rs}}=\left(\widetilde{\mathbf{X}}_{\mathrm{rot}}, \widetilde{\mathbf{X}}_{\mathrm{s}}\right)
$$

into generators $\widetilde{\mathbf{X}}_{\text {rot }}$ of rotations about the center of mass and a generator $\widetilde{\mathbf{X}}_{\mathrm{s}}$ of scalings about the center of mass. Then we have that

$$
\widetilde{\mathfrak{J}}_{\mathrm{rs}}=\operatorname{vol}\left(\widetilde{\mathbf{X}}_{\mathrm{rs}}\right)=\operatorname{vol}\left(\widetilde{\mathbf{X}}_{\mathrm{rot}}\right) \cdot \operatorname{vol}\left(\widetilde{\mathbf{X}}_{\mathrm{s}}\right),
$$

since $\widetilde{\mathbf{X}}_{\mathrm{s}}$ is orthogonal to $\widetilde{\mathbf{X}}_{\text {rot }}$.

Fourth, we have that

$$
\operatorname{vol}\left(\widetilde{\mathbf{X}}_{\mathrm{s}}\right)=\mathrm{L}
$$


up to a constant, independent of $\boldsymbol{q}$, where $\mathrm{L}=\mathrm{L}(\boldsymbol{q})$ is given by equation (9). To see this, note that the effect of a scaling at $\boldsymbol{q}$ about the center of mass is proportional to the value of $\mathrm{L}$ at $\boldsymbol{q}$. As for the other volume element in (120), we have

$$
\operatorname{vol}\left(\widetilde{\mathbf{X}}_{\text {rot }}\right)=\sqrt{\operatorname{det} M}
$$

where $\mathrm{M}$ is the tensor of inertia of the configuration $\boldsymbol{q}$ about the center of mass, whose matrix elements with respect to an orthogonal cartesian system $x, y, z$ are given by equation (12). This formula for the volume element is presumably standard. A way to see how it comes about is the following.

To simplify the notations, let us drop "tildas" and "rot" and stipulate that in this paragraph $\left(\mathbf{X}_{i}\right), i=$ $x, y, z$, denotes a basis for the generators of rotations about the center of mass $\overrightarrow{\mathrm{q}}_{\mathrm{cm}}(x, y, z$ refer to any orthogonal frame with origin in the center of mass). Then the volume element in 122 is given by $\sqrt{\operatorname{det} A}$, where $\mathrm{A}$ is the matrix with entries $A_{i j}=g_{e}\left(\mathbf{X}_{i}, \mathbf{X}_{j}\right)$. For $\widetilde{\boldsymbol{q}}=\boldsymbol{q}-\overrightarrow{\mathrm{q}}_{\mathrm{cm}}$ a configuration relative to the center of mass, let $\widetilde{\boldsymbol{q}}=\left(\vec{q}_{1}, \ldots, \vec{q}_{N}\right)$. Observe that a generator corresponding to the action of a rotation on configurations is of the form $\mathbf{X}_{\boldsymbol{\Omega}}(\widetilde{\boldsymbol{q}})=\left(\boldsymbol{\Omega} \times \vec{q}_{1}, \ldots, \boldsymbol{\Omega} \times \vec{q}_{N}\right)$, where $\boldsymbol{\Omega}$ is a 3-dimensional vector of components $\Omega^{i}$ (with respect to the $x y z$ frame). Thus the 3 -dimensional Lie algebra corresponds naturally to the 3 -dimensional vectors $\boldsymbol{\Omega}$, with $\Omega^{i}$ being the coordinates of a general element $\mathbf{X}_{\boldsymbol{\Omega}}$ of the Lie algebra with respect to the basis $\left(\mathbf{X}_{i}\right)$. A vector $\boldsymbol{\Omega}$ corresponds to a general instantaneous rotational motion. Consider the kinetic energy

$$
K=\frac{1}{2} g_{e}\left(\mathbf{X}_{\boldsymbol{\Omega}}, \mathbf{X}_{\boldsymbol{\Omega}}\right)
$$

for such a motion. On the one hand, it is known to be given by

$$
K=\frac{1}{2} M_{i j} \Omega^{i} \Omega^{j}
$$

where $\mathrm{M}=\left\{M_{i j}\right\}$ is the moment of inertia tensor. On the other hand, expanding the right hand side of $(123)$ by expressing $\mathbf{X}_{\boldsymbol{\Omega}}=\sum_{i} \Omega^{i} \mathbf{X}_{i}$ in the basis $\left(\mathbf{X}_{i}\right)$, one obtains

$$
g_{e}\left(\mathbf{X}_{\boldsymbol{\Omega}}, \mathbf{X}_{\boldsymbol{\Omega}}\right)=g_{e}\left(\mathbf{X}_{i}, \mathbf{X}_{j}\right) \Omega^{i} \Omega^{j}=A_{i j} \Omega^{i} \Omega^{j}
$$

Thus equating the right hand sides of 123 and 124 , one sees that the matrix $A$ is indeed the tensor of inertia $\mathrm{M}$, whence equation 122.

Fifth (and finally), substituting in (114) the formula for $\mathfrak{J}_{e}$ given by (115), with $\operatorname{vol}\left(\mathbf{X}_{V}\right)=\mathfrak{J}_{\text {rs }}$, and using formulas 120,121 , and 122 for $\widetilde{\mathfrak{J}}_{\text {rs }}$, we have that

$$
\mathfrak{J}=\mathrm{L} f^{7 / 2} \sqrt{\operatorname{det} \mathrm{M}},
$$

which is formula (58) for the shape Jacobian.

\section{G. More Gauge Freedom}

As we have already stressed, the lift to $\mathcal{Q}$ of the Laplace-Beltrami operator $\Delta_{B}$ on $\mathcal{Q}$ is by no means unique. The "canonical lift" 65 , with $\mathfrak{J}$ given by equation (58), is very natural, but other choices are possible. This lack of uniqueness increases the gauge freedom we have in defining the Schrödinger gauge. In particular, we may use this freedom to define a shape Jacobian that is an invariant function on $\mathbf{Q}$, i.e., a function of the configuration $\boldsymbol{q}$ which depends only on its shape.

Note that $\mathfrak{J}$ is not invariant: $f^{7 / 2}$ scales like $\mathrm{L}^{-7}$ and $\sqrt{\operatorname{det} \mathrm{M}}$ like $\mathrm{L}^{3}$. We thus have that

$$
\mathfrak{J}=\mathrm{L}^{-3} \mathfrak{J}_{B}
$$

where

$$
\mathfrak{J}_{B}=f_{1}^{7 / 2} \sqrt{\operatorname{det} \mathrm{M}_{1}}
$$

is invariant. Here the subscript 1 indicates the quantities have to be evaluated, not at $\boldsymbol{q}$, but at $\boldsymbol{q}_{1}$, the configuration with $\mathrm{L}=1$ obtained by rescaling $\boldsymbol{q}$.

To define a lift $\widehat{\Delta}_{B}$ of $\Delta_{B}$, we could as well have used the invariant

$$
\mathfrak{J}_{B}=\mathrm{L}^{3} \mathfrak{J}=\mathrm{L}^{4} f^{7 / 2} \sqrt{\operatorname{det} \mathrm{M}}
$$

instead of $\mathfrak{J}$. This would have in no way affected the results and the arguments in Sect. VIC and Sect. VIE, though it would yield somewhat different potentials $V$ and $U$ in 52 and 63 , respectively.

\section{H. The Canonical Conformal Factor}

Instead of computing $\mathfrak{J}_{B}$ for a given $f$, we might read 127) the other way round, and ask what is the conformal factor that gives rise to the simplest $\mathfrak{J}_{B}$. The simplest possibility is $\mathfrak{J}_{B}=1$ and this is associated with $f(\boldsymbol{q}) \equiv$ $f_{c}(\boldsymbol{q})$ given by equation 13 , i.e.,

$$
f(\boldsymbol{q})=f_{c}(\boldsymbol{q}) \equiv \mathrm{L}^{-\frac{8}{7}}(\operatorname{det} \mathrm{M})^{-\frac{1}{7}}
$$

the canonical conformal factor. Note that replacing $\mathfrak{J}$ in (56) with $\mathfrak{J}_{B}=1$ gives $V_{1}=0$ so that the potential in the Hamiltonian (52) is $V=V_{2}$, with the form (57) of $V_{2}$ unaffected. (Of course, one needs to evaluate it for $f=f_{c}$.) Similarly, the potential $U$ in 64 becomes

$$
U=-f_{c} \mathscr{E}-\frac{\hbar^{2}}{8} \frac{n-2}{n-1} f_{c} R_{g_{c}},
$$

where $R_{g_{c}}$ (cf. (91) is now the scalar curvature of the metric $g=g_{c}$ associated with $f_{c}$. 


\section{SUBSYSTEMS}

\section{A. Conditional Wave Functions}

In physics we are usually concerned not with the entire universe but with subsystems of the universe, for example with a hydrogen atom or a pair of entangled photons. The quantum mechanical treatment of such systems involves the quantum state of that system, often given by its wave function - not the wave function of the universe. Bohmian mechanics provides a precise formulation and understanding of this notion in terms of the conditional wave function 11

$$
\psi(x)=\Psi(x, Y),
$$

where $\Psi=\Psi(q)=\Psi(x, y)$ is the wave function of the universe, with $x$ and $y$ the generic variables for the configurations of the system and its environment, respectively, and where $Y$ is the actual configuration of the environment. The conditional wave function of a Bohmian system behaves exactly as one would expect the wave function of a system to behave, with respect to both dynamics and statistics. It is natural to ask how and whether the conditional wave function can be defined for Bohmian mechanics on shape space.

For this the following problem arises. There is no natural product structure

$$
\mathcal{Q}=\mathcal{Q}_{\text {sys }} \times \mathcal{Q}_{\text {env }}
$$

for shape space: Here the system is a collection of (labelled) particles with its own shape space $\mathscr{Q}_{\text {sys }}=\mathcal{X}$, the set of possible shapes $X$ of the system, and the environment consists of the rest of the particles of the universe, with shape space $\mathcal{Q}_{e n v}=\mathcal{Y}=\left\{Q_{e n v}=Y\right\}$, with $Y$ the shape associated with the particles (labels) of the environment. The crucial fact is that it is not true that

$$
2=x \times y .
$$

$X$ and $Y$ don't involve sufficient information to determine the complete shape $Q$. What is missing is the spatial relationship between these shapes.

Nonetheless we have that $\mathcal{Q}$ can be identified with

$$
x_{Y} \times_{y} y=\left\{(X, Y) \mid Y \in \mathcal{Q}_{e n v}, X \in \mathcal{Q}_{Y}\right\},
$$

where

$$
\mathscr{Q}_{Y}=\left\{Q \in \mathcal{Q} \mid Q_{e n v}=Y\right\} .
$$

We may then define the conditional wave function for the subsystem, for $Y \in \mathcal{Q}_{e n v}$ and universal wave function $\Psi$, by

$$
\psi(x)=\Psi(x, Y), \quad x \in \mathcal{Q}_{Y} .
$$

This looks like the usual conditional wave function, but it is important to bear in mind that, unlike with the usual conditional wave function, here $x$ represents the shape of the universe for a fixed $Y$ and there are no obvious natural coordinates to efficiently describe it.

To obtain such a thing the notion of a frame might be useful: Given $Y \in \mathcal{Q}_{e n v}$, a frame $\boldsymbol{Y}$ is a choice of point in the fiber over $Y$ in the absolute configuration space of the environment. ( $\boldsymbol{Y}$ corresponds roughly to the usual notion of frame of reference.)

Given $Y$ and a frame $\boldsymbol{Y}$, we obtain natural coordinates for $\mathcal{Q}_{Y}$ : Given $X \in \mathcal{Q}_{Y}$ there is a unique $\boldsymbol{X} \in \mathbb{R}^{3 M}$ (with $M$ the number of particles of the subsystem, which we shall assume from here on is such that the number of particles in the environment $N-M \geq 3$ ) such that $(\boldsymbol{X}, \boldsymbol{Y})$ is in the fiber above $X \in \mathcal{Q}_{Y}$. The map $X \mapsto \boldsymbol{X}$ is a one-to-one correspondence between $\mathscr{Q}_{Y}$ and $\mathbb{R}^{3 M}$.

Given the frame $\boldsymbol{Y}$, we may represent the conditional wave function by

$$
\widehat{\psi}(\boldsymbol{x})=\psi(x)
$$

where $x$ corresponds to $\boldsymbol{x}$. In other words,

$$
\widehat{\psi}(\boldsymbol{x})=\widehat{\Psi}(\boldsymbol{x}, \boldsymbol{Y})
$$

with $\widehat{\Psi}$ the lift of $\Psi$ to absolute configuration space, $\boldsymbol{Y}$ a lift of $Y, \boldsymbol{x}$ the lift of $x$ in the frame $\boldsymbol{Y}$, and the "hat" refers to any of the gauge equivalent representations $\widehat{\Psi}_{1}$, $\widehat{\Psi}_{2}, \widehat{\Psi}_{3}$ (and presumably $\widehat{\Psi}_{S}$ ) of the universal wave function that we have described in Sect. VIC. Thus, the absolute configuration space level conditional wave functions $\widehat{\psi}_{1}, \widehat{\psi}_{2}$ and $\widehat{\psi}_{3}$ (and $\widehat{\psi}_{S}$ ) are at the same time different representations of the shape space conditional wave function. Moreover, the $\widehat{\psi}$ s obtained using different frames are in an appropriate sense equivalent.

This conditional wave function $\widehat{\psi}$ behaves like the wave function of the subsystem, both with respect to the dynamics of configurations, via the guiding equation, and with respect to probabilities for the subsystem, via what has been called the fundamental conditional probability formula [1]. With regard to the dynamics, this is clear from the form of the dynamics on absolute configuration space. The latter, while true, is not at all so clear. That it is so follows from the analysis in Sect. VIII

\section{B. Subsystems and the Role of Projectivity}

The time-parameter corresponding to the use of the denominator in footnote 3 has the nice feature that the dynamics using that time-parameter depends on fewer details of the wave function than would be the case if the denominator were deleted: with the denominator the dynamics depends only on the ray of $\psi$, with $\psi$ and $c \psi$ yielding the same dynamics for any constant $c \neq 0$. This has a particularly nice implication for the behavior of subsystems. With this choice of time-parameter the dynamics for a subsystem will often not depend upon the configuration of its environment, with the subsystem evolving 
according to an autonomous evolution involving only the configuration and the (conditional) wave function of the subsystem itself [11. This would happen when the subsystem is suitably decoupled from its environment, for example for a product wave function when there is no interaction between system and environment. Without the denominator this would not be true, and there would appear to be an additional nonlocal dependence of the behavior of a subsystem on that of its environment that would not be present with a time-parameter associated with the use of the usual denominator.

\section{The Emergence of Metrical Time}

By metrical time we refer to any objective physical coordination of the configurations along a geometrical path in a configuration space with the points of a onedimensional continuum: a (continuous) mapping from the continuum onto the path. The continuum is usually represented by the real numbers, but it need not be. However, it should be physically distinct from the particular continuum that is the path itself.

Understood in this way, metrical time does not exist, from the relational point of view, for the universe as a whole. However, for subsystems of the universe metrical time naturally emerges: the continuum with which the geometrical path corresponding to the evolution of the subsystem is coordinated can be taken to be the path of its environment, with the obvious mapping between the paths.

\section{PROBABILITY}

\section{A. Bohmian Mechanics and Probability}

In Bohmian mechanics, for a non-relativistic system of particles, the configuration of a system is regarded as random, with randomness corresponding to the quantum equilibrium distribution $\mu^{\psi}$ given by $|\psi|^{2} d q$. What this actually means, in a deterministic theory such as Bohmian mechanics, is a delicate matter, involving a long story [11] with details and distinctions that we shall ignore here. However a crucial ingredient for that analysis - for an understanding of the origin of quantum randomness in a universe governed by Bohmian mechanics - is the fundamental conditional probability formula for the conditional distribution of the configuration $X_{t}$ of a system at time $t$ given that of its environment $Y_{t}$ at that time:

$$
P^{\Psi_{0}}\left(X_{t} \in d x \mid Y_{t}\right)=\left|\psi_{t}(x)\right|^{2} d x,
$$

where $\Psi_{0}$ is the initial wave function of the universe and $P^{\Psi_{0}}$ is the probability distribution on trajectories arising from the Bohmian dynamics with an initial quantum equilibrium distribution, and $\psi_{t}$ is the (normalized) conditional wave function 129 of the system at time $t$.
A crucial ingredient in proving (133) is equivariance [11: if at any time the system configuration is randomly distributed according to $\left|\Psi_{0}\right|^{2}$, then at any other time $t$ it will be distributed according to $\left|\Psi_{t}\right|^{2}$. Equivariance is an immediate consequence of the continuity equation arising from the Schrödinger equation:

$$
\frac{\partial \rho^{\Psi}}{\partial t}+\operatorname{div} J^{\Psi}=0
$$

with $\rho^{\Psi}=|\Psi|^{2}$, the quantum equilibrium distribution, and

$$
J^{\Psi}=\rho^{\Psi} v^{\Psi},
$$

the quantum probability current, where $v^{\Psi}$ is the Bohmian velocity in the right hand side of 22,

$$
v^{\Psi}=\hbar \operatorname{Im} \frac{\nabla \Psi}{\Psi}
$$

(as usual here, the masses are absorbed in the gradient).

More generally, for Bohmian mechanics on a Riemannian manifold with metric $g$, the quantum equilibrium distribution $\mu^{\Psi}$ is given by $|\Psi|^{2} d \mu_{g}$. In terms of any coordinate system $x=\left(x_{1}, \ldots, x_{n}\right)$, we have that

$$
d \mu_{g}=\sqrt{|g|} d x
$$

where $d x=d x_{1} \cdots d x_{n}$ and $g=g_{i j}=g\left(\mathbf{X}_{i}, \mathbf{X}_{j}\right)$, with $\mathbf{X}_{i}=\partial / \partial x_{i}$.

Moreover, as already indicated several times, the Bohmian velocity on a Riemannian manifold is still given by 136 with $\nabla=\nabla_{g}$, the gradient with respect to the metric $g$. Furthermore, letting $\rho^{\Psi}$ be the density of $\mu^{\Psi}$ with respect to $\mu_{g}$, i.e., $\rho^{\Psi}=|\Psi|^{2}$, the current is most conveniently represented as a density with respect to $\mu_{g}$ as in (135); in this representation the continuity equation 134) holds for $\operatorname{div}=\operatorname{div}_{g}$, the divergence with respect to $g$. So, all the Bohmian structure, including the quantum equilibrium measure, transfers straightforwardly from the familar Euclidean setting to a general Riemannian manifold.

\section{B. The Problem of Non-Normalizable Measures}

Note that since it is translation and scaling invariant, the wave function in the Schrödinger gauge or in any of the gauges discussed in Sect VIB are not normalizable. In other words, $\mu^{\widehat{\Psi}_{S}}$ and $\mu^{\Psi}$ given by 100 and (96), respectively, are non-normalizable. However, since the non-normalizability arises from unobservable (and, from a shape space point of view, unphysical) differences and dimensions it should somehow not be a problem.

Nonetheless, the real question is how the empirical distributions arising from the fundamental shape space level compare with those coming from the physics in a gauge. While the different gauges, such as the Schrödinger 
gauge, correspond to theories that, we argued, are empirically equivalent to the fundamental shape space theory, that was only in purely dynamical terms. We have not yet addressed the possible differences in empirical distributions that may arise. We would like to see that they don't.

There are several considerations that suggest that the non-normalizability should not be a genuine problem:

1) As just mentioned, the non-normalizability arises only from non-observable dimensions, suggesting that it should be physically irrelevant.

2) It is the universal wave function $\widehat{\Psi}$ (in any of the gauges) that is not normalizable. But the universal wave function is rarely used in practice. In quantum mechanics we usually deal, not with the entire universe, but with small subsystems of the universe. The wave functions with which we usually deal are thus conditional wave functions, and there seems to be no reason why these should fail to be normalizable.

3) In statistical mechanics the Lebesgue measure on the phase space for a gas in a box is nonnormalizable. Why is this not a problem? It is because the energy is a constant of the motion, and the restriction of the Lebesgue measure to the energy surface is normalizable (for suitable potentials). Similarly here, with Bohmian mechanics in the Schrödinger gauge, or in any of the gauges on absolute configuration space, the center of mass and the moment of inertia about the origin, or about the center of mass, are constants of the motion. Thus, it would seem that the appropriate measure that we should be considering here is $\mu_{\Gamma}^{\widehat{\Psi}}$, the conditional distribution for $\mu^{\widehat{\Psi}}$ given $\Gamma$, the one given by $|\widehat{\Psi}|^{2}$ on a surface $\Gamma$ of constant center of mass and moment of inertia about the origin, and not on the entire absolute configuration space, and this is presumably normalizable. Moreover, all such probability distributions, for different choices of $\Gamma$, are physically equivalent, since they correspond to the same probability distribution on shape space.

The probability distributions described in 3), while they seem to correspond to the appropriate measures on shape space, appear to be entirely inappropriate insofar as the fundamental conditional probability formula is concerned. For example, for a single-particle system the configuration of that system would be completely determined by the configuration of its environment, rather than being randomly distributed according to the quantum equilibrium distribution. What gives?

\section{The Fundamental Conditional Probability Formula for Evolving Wave Functions}

In order to answer the last question, as well as to obtain a sharp resolution of the non-normalizability issue and an understanding of how to carry out the usual quantum equilibrium analysis [1] yielding the Born rule, the following observation is crucial: The conditional distribution, arising from $\mu^{\widehat{\Psi}}$, of the configuration of a system given its environment in absolute configuration space is unphysical, and is thus not relevant to an appropriate quantum equilibrium analysis. That is because (from the shape space point of view) the absolute configuration of the environment is unphysical. What is physical, and what we should be conditioning on, is the shape of the environment. And when we condition on this, the result will be given in terms of the conditional wave function $\widehat{\psi}$ 131.

In fact, on the fundamental level, with respect to the quantum equilibrium distribution on shape space, $d \mu_{B}^{\Psi}=|\Psi|^{2} d \mu_{B}$ (where $d \mu_{B} \equiv d \mu_{g_{B}}$ ), the conditional distribution of the configuration of a system (i.e., of the configuration of the universe, see (130) given the shape of its environment is perhaps most naturally expressed on the absolute level, via the use of a frame and of the corresponding conditional wave function $\widehat{\psi}$ in any one of the first three gauges - for example, the conditional wave function in the first gauge we considered, namely the straightforward lift (45) - using the frame $\boldsymbol{Y}$ rather than the shape $Y$. And since the quantum equilibrium distribution for this conditional wave function represents the corresponding conditional distribution on shape space (as we shall argue below), it follows that the conditional wave function on absolute configuration space is normalizable.

That this is in fact so, i.e., that the lift to absolute configuration space of the fundamental conditional probability formula on shape space is given by the Born rule for the conditional wave function on absolute configuration space, can be seen as follows:

- Unlike marginal distributions, conditional measures are well defined, up to a constant multiple, even for a non-normalizable measure $\mu$.

- Suppose we condition on something, for example, the environment $\boldsymbol{Y}$ of a subsystem, corresponding to a leaf $\Gamma_{\mathscr{E}}$ of a foliation $\mathscr{E}$ (e.g., into the level sets of the random variable $\boldsymbol{Y}$ ). We would obtain the same result if we had first conditioned on a leaf $\Gamma_{\mathscr{F}}, \Gamma_{\mathscr{E}} \subset \Gamma_{\mathscr{F}}$, of a coarser foliation $8 \mathscr{F} \subset \mathscr{E}$, obtaining the conditional measure $\mu_{\Gamma_{\mathscr{F}}}$, and then, with respect to $\mu_{\Gamma_{\mathscr{F}}}$, conditioned on $\Gamma_{\mathscr{E}}$.

\footnotetext{
8 identifying $\mathscr{F}$ and $\mathscr{E}$ with the $\sigma$-algebras they generate, consisting of the measurable sets that are unions of the leaves of the respective foliations
} 
- For $\mu=\mu^{\widehat{\Psi}}$, the (non-normalizable) quantum equilibrium distribution on absolute configuration space, we can choose $\mathscr{F}$ so that the measures $\mu_{\Gamma_{\mathscr{F}}}$ all correspond to the quantum equilibrium distribution on shape space, and $\mathscr{E}$ so that it corresponds to the shape of the environment.

- If this can indeed be done we obtain our desired result.

In more detail, fix now the subsystem, and let $\mathscr{E}$ correspond to the configuration $\boldsymbol{Y}$ of its environment. $\mathscr{F}$ must be chosen so that the following is true: Each leaf of $\mathscr{F}$ must provide a representation of shape space as a measure space. Not only must there be a smooth bijection between shape space and each leaf of $\mathscr{F}$, but under this bijection we must have that each of the measures $\mu_{\Gamma_{\mathscr{F}}}$ corresponds to $\mu_{B}^{\Psi}$. Moreover, we must also have that (i) the leaf of $\mathscr{F}$ to which an absolute configuration belongs is determined by the configuration of its environment, i.e., $\mathscr{F} \subset \mathscr{E}$, and that (ii) different leaves of $\mathscr{E}$ belonging to the same leaf of $\mathscr{F}$ correspond to environmental configurations with different shapes, so that with respect to the bijection, the configuration of the environment corresponds to its shape.

Such an $\mathscr{F}$ can be generated from a cross-section of the absolute configuration space of the environment regarded as a bundle over its shape space. Such a crosssection naturally induces a cross-section in the universal absolute configuration space regarded as a bundle over shape space (since for any shape there is a unique absolute configuration compatible with the environmental cross-section). The cross-section so obtained provides a single leaf $\Sigma_{1}$ of $\mathscr{F}$; the other leaves of $\mathscr{F}$ are obtained by the application of the symmetry group $G$ to $\Sigma_{1}$. In this way, absolute configuration space can be identified with $G \times \Sigma_{1}$, with $\mathscr{F}$ corresponding to $G$, i.e., having leaves $\Sigma_{\mathrm{g}}=\mathrm{g} \Sigma_{1}$.

Note that with this $\mathscr{F}$ we have, essentially by construction, that the shape of the environment corresponds, on each leaf of $\mathscr{F}$, to its absolute configuration. Note also that this would not be true for the foliation corresponding to the (quotient under rotations of the) surfaces $\Gamma$ described in 3) of Sect. VIIIB, for which a single shape of the environment would correspond to many different absolute environmental configurations on each leaf (even after rotations have been factored out).

We now check that for this $\mathscr{F}$ the conditional measures $\mu_{\Gamma_{\mathscr{F}}}$ correspond to the quantum equilibrium distribution on shape space. For this we will use the following general formula for the Riemannian volume element $\mu_{g}$ in terms of a general basis of vector fields $\mathbf{X}_{i}$ :

$$
d \mu_{g}=\sqrt{|g|} d \omega_{1} \cdots d \omega_{n}
$$

where $|g|$ is defined below 102 . This formula is similar to 137 for the case when the vector fields are coordinate vector fields, but with $d x_{1} \ldots d x_{n}$ replaced by $d \omega_{1} \cdots d \omega_{n}$, the volume element arising from the $n$-form $d \omega_{1} \wedge \cdots \wedge d \omega_{n}$, where $\omega_{1}, \ldots, \omega_{n}$ is the basis of 1 -forms dual to the basis $\mathbf{X}_{1}, \ldots, \mathbf{X}_{n}$ of vector fields, see Sect. VIE

For the basis of vector fields $\mathbf{X}=\left(\mathbf{X}_{V}, \mathbf{X}_{H}\right)$ described there, we obtain that

$$
d \mu_{g}=\sqrt{|g|} d \omega_{V} d \omega_{H}=\sqrt{\left|g_{V}\right|} \sqrt{\left|g_{H}\right|} d \omega_{V} d \omega_{H}
$$

where $d \omega_{V}$ is the volume element arising from $\mathbf{X}_{V}$, a volume element on the fibers of absolute configuration space, and $d \omega_{H}$ corresponds to the volume element on shape space arising from the coordinate system involved in the definition of $\mathbf{X}_{H}$.

Since $\sqrt{\left|g_{H}\right|} d \omega_{H}$ corresponds to $\sqrt{\left|g_{B}\right|} d x=d \mu_{B}$, where $d x$ is the coordinate measure on shape space for these coordinates, we have that $d \mu_{g}$ corresponds to $\sqrt{\left|g_{V}\right|} d \omega_{V} d \mu_{B}$. Moreover, $d \omega_{V}$ is, up to a constant factor, the image of the right Haar measure $\mu_{G}$ on $G$. We thus have, using the representation $G \times \Sigma_{1}$ for absolute configuration space, that

$$
d \mu_{g}=\mathfrak{J} d \mu_{G} d \mu_{B}^{1},
$$

where $\mathfrak{J}$ is given in 113 and $\mu_{B}^{1}$ is the image of $\mu_{B}$ on $\Sigma_{1}$.

Now since $\widehat{\Delta}_{B}$, see Sect. VIB is self-adjoint, not with respect to $d \mu_{g}$, but with respect to $\mathfrak{J}^{-1} d \mu_{g}=d \mu_{G} d \mu_{B}^{1}$, we have that, for $\widehat{\Delta}_{B}$, and any wave function $\Psi$ on shape space, the quantum equilibrium distribution $\mu^{\Psi}$ is given by

$$
d \mu^{\widehat{\Psi}}=|\widehat{\Psi}|^{2} d \mu_{G} d \mu_{B}^{1}
$$

Thus, for $\mu^{\widehat{\Psi}}$, the conditional probability distribution given $G$ is $|\widehat{\Psi}|^{2} d \mu_{B}^{1}$, i.e., the image of $\mu_{B}^{\Psi}$, just as we wanted.

Finally, as we indicated earlier in Sect. VID as we proceed through the first three gauges, each involving its own wave function and measure for self-adjointness, the transformations connecting the gauges have been so defined as to leave invariant the corresponding quantum equilibrium distributions $\mu^{\widehat{\Psi}}$. For each gauge, we are in fact dealing with the same measure on absolute configuration space, and hence the same conditional measure given a leaf $\Gamma_{\mathscr{F}}$ of the foliation $\mathscr{F}$ and the same conditional measure given a leaf $\Gamma_{\mathscr{E}}$ of the foliation $\mathscr{E}$. Thus in each gauge, the conditional distribution of the quantum equilibrium measure given the configuration of the environment yields, in fact, the conditional distribution on shape space given the shape of the environment, which is what we wanted to establish.

In summary, we have obtained that the conditional distribution of the configuration $X$ of a system given the shape $Y$ of its environment is given by

$$
\mu_{B}^{\Psi}(X \in d x \mid Y)=\mu^{\widehat{\Psi}}(\boldsymbol{X} \in d \boldsymbol{x} \mid \boldsymbol{Y}) .
$$

As a consequence, we have (from equivariance) the fundamental conditional probability formula on shape space, 
for the conditional distribution of the configuration $X_{t}$ of a system at time $t$ given the shape $Y_{t}$ of its environment at that time.

\section{Fundamental conditional probability formula on shape space:}

$$
P^{\Psi_{0}}\left(X_{t} \in d x \mid Y_{t}=Y\right)=\mu^{\widehat{\Psi}_{t}}(\boldsymbol{X} \in d \boldsymbol{x} \mid \boldsymbol{Y}),
$$

where $\Psi_{0}$ is the initial universal wave function on shape space (at $t=0), \boldsymbol{Y}$ is a lift of $Y, \boldsymbol{x}$ the lift of $x$ in the frame $\boldsymbol{Y}, \Psi_{t}$ is the universal wave function at time $t$, with $\mu^{\widehat{\Psi}_{t}}$ the non-normalizable quantum equilibrium measure on absolute configuration space - the lift (96) of the quantum equilibrium distribution $\mu_{B}^{\Psi}$ on shape space.

In the 3-gauge this can be written in a more familiar and explicit manner:

$$
\begin{aligned}
P^{\Psi_{0}}\left(X_{t} \in d x \mid Y_{t}=Y\right) & =\left|\widehat{\psi}_{3, t}(\boldsymbol{x})\right|^{2} d \boldsymbol{x} \\
& =C\left|\widehat{\Psi}_{3, t}(\boldsymbol{x}, \boldsymbol{Y})\right|^{2} d \boldsymbol{x},
\end{aligned}
$$

where $\widehat{\psi}_{3, t}$ is the (normalized) conditional wave function in the 3 -gauge in the frame $\boldsymbol{Y}$ and $C$ is a normalization constant.

\section{The Physical Significance of the Conditional Distribution for Stationary Wave Functions}

However, what has just been said is not quite right for the Schrödinger gauge corresponding to the transition from $(52)$ to $(63)$. As already indicated at the end of Sect. VID this involves no change of measure for selfadjointness, so that $\mu^{\widehat{\Psi}_{S}} \neq \mu^{\widehat{\Psi}}$. This might seem bad. On the other hand, the change in $\mu^{\widehat{\Psi}}$ is precisely the one implied by the random time change arising from replacing $f^{-1} \nabla$ by $\boldsymbol{\nabla}$, as described above (61). This seems sort of good. But one should be puzzled by the fact that this random time change leads to a change in the measure $\mu^{\widehat{\Psi}}$, which would seem to have some physical significance. But how could it, since the random time change has no physical significance?

So there are several questions here that need to be understood better: (i) What is genuinely physically significant in $\mu^{\widehat{\Psi}}$ ? (ii) How does that resolve the apparent problem that in the Schrödinger gauge we are dealing with a $\mu^{\widehat{\Psi}_{S}}$ that is incompatible with $\mu_{B}^{\Psi}$ and thus apparently one that would yield an incompatible fundamental conditional probability formula in the Schrödinger gauge?

A crucial ingredient in an answer to these questions is the claim that it is not exactly $\mu^{\widehat{\Psi}}$, resp. $\mu^{\widehat{\Psi}_{S}}$, that is physically relevant. Rather what is physically relevant is the associated current $J^{\widehat{\Psi}}=\rho^{\widehat{\Psi}} v^{\widehat{\Psi}}$, resp. $J^{\widehat{\Psi}_{S}}=\rho^{\widehat{\Psi}_{S}} v^{\widehat{\Psi}_{S}}$, where $\rho^{\widehat{\Psi}} \propto \rho^{\widehat{\Psi}_{3}}$, resp. $\rho^{\widehat{\Psi}_{S}}$, is the density of $\mu^{\widehat{\Psi}}$, resp. $\mu^{\widehat{\Psi}_{S}}$, with respect to the Lebesgue measure. The current is invariant under all the transitions, either because both factors are or because the changes in the factors compensate each other.

Now why should the current be what is physically relevant? Because it yields the same crossing probabilities for hypersurfaces - that either yield the probability distribution on geometrical paths in configuration space, or the probability distribution associated with the return map for Poincaré sections corresponding to physical situations on which we wish to condition. Concerning the former, this could correspond to conditioning on the value of a suitable clock variable, for which the corresponding conditional wave functions have more familiar quantum evolutions, so that first conditioning on such a clock variable would put us back in a more familiar situation to which the argument described above would apply.

Be that as it may, let's return to the question of why the change in the measure $\mu^{\widehat{\Psi}}\left(\mu^{\Psi_{S}}=f^{-1} \mu^{\widehat{\Psi}}\right)$ arising from the random time change has no physical significance. We have argued that the main physical relevance of $\mu^{\widehat{\Psi}}$ resides in the implied conditional distribution of a subsystem given its environment. Suppose $f$ depends only upon the environment. In this case, the change in measure associated with $f$ produces no change in the corresponding conditional distribution 9 And it seems likely that for reasonable choices of $f$, such as those given in Sect.IID above, it will approximately be a function of the environment, with negligible error for subsystems of reasonable size, much smaller than that of the universe.

But even if this is so, the question remains as to exactly what of physical significance this conditional distribution represents. After all, the transition to the Schrödinger gauge required relational time, but if we take relational time seriously, what is physical is not the configuration $Q_{t}$ of the universe at some time $t$, but the geometrical path of the full history of the configuration, with no special association of the configurations along a path with times. In this (more physical) framework, the conditional distribution of the configuration $X_{t}$ of a subsystem given the configuration $Y_{t}$ of its environment is not meaningful.

\footnotetext{
${ }^{9}$ Letting $\boldsymbol{x}$ and $\boldsymbol{y}$ be the configuration variables of system and environment, respectively, note that if $f$ does not depend on $\boldsymbol{x}$, the kinetic energy term in the Hamiltonian 84 will be

$$
-\frac{\hbar^{2}}{2}\left[\frac{1}{f}\left(\boldsymbol{\nabla}^{2}\right)_{\boldsymbol{x}}+\left(\boldsymbol{\nabla} \cdot \frac{1}{f} \boldsymbol{\nabla}\right)_{\boldsymbol{y}}\right],
$$

where $\boldsymbol{\nabla}$ and $\boldsymbol{\nabla} \cdot$ are the mass-weighted Euclidean gradient and divergence and the subscripts refer to their restrictions to the $\boldsymbol{x}$ variables and the $\boldsymbol{y}$-variables, respectively. Accordingly, whenever the system is decoupled from its environment, its conditional wave function $\widehat{\psi}_{3}(\boldsymbol{x})=\widehat{\Psi}_{3}(\boldsymbol{x}, \boldsymbol{Y})$ will evolve (after suitable rescaling) according to the standard Schrödinger Hamiltonian with masses $f(\boldsymbol{Y}) m_{\alpha}, \alpha=1, \ldots M$, where $\boldsymbol{Y}$ is the actual configuration of the environment. Thus, in this case, the effect of the environment on the system corresponds just to a (possibly time-dependent) rescaling of the masses. Then it turns out that the 3-gauge is more similar to the Schrödinger gauge than one may have expected.
} 
What is meaningful is $(\mathrm{i})$ a probability distribution $\mathbb{P}$ on the space $\mathscr{P}$ of (geometrical, i.e. unparametrized) paths (determined by the current, for example by using a cross-section, as we shall explain in Sect. VIIIE and (ii) the conditional distribution relative to $\mathbb{P}$ of the configuration $X_{Y}$ of the subsystem when the path $\gamma \in \mathscr{P}$ has environmental configuration $Y$, given that the path passes through a configuration with environment $Y, Y \in \gamma 10$ We assume here that there is at most one such configuration for (any) $Y{ }^{11}$

\section{E. The Association Between Measures on Path Space and on Configuration Space}

We shall now elaborate on the relations between measures on a configuration space $\mathscr{Q}$ and measures on a set $\mathscr{P}$ of (smooth) paths on $\mathscr{Q}$ forming a one-dimensional foliation of $\mathscr{Q}$. First, let us fix the notations: we shall denote by $\gamma$ a path in $\mathscr{P}$, by $q$ a point in $\mathscr{Q}$ and by $\gamma(q)$ the path passing through $q$.

We introduce the notion of time function on $\mathscr{Q}$ : a smooth real-valued function $\tau=\tau(q), q \in \mathscr{Q}$, which is monotonic (and extends from $-\infty$ to $+\infty$ ) on each path $\gamma \in \mathscr{P}$. The latter is related to, but different from, the notion of dynamics on $\mathscr{Q}$ (in the sense of the theory of dynamical systems): a one parameter family of (invertible and smooth) maps $T=T_{s}$ on $\mathscr{Q}$, with time $s$ varying on the reals. Clearly, a time function $\tau$ generates a dynamics $T=T^{\tau}$ such that

$$
\tau\left(T_{s}(q)\right)=\tau(q)+s .
$$

Let $\tau$ be a time function and denote by $d \tau_{\gamma}(q)$ the infinitesimal increment of time (with respect to the time function $\tau$ ) along a path $\gamma$ passing through $q$. Consider the function on path space (random variable) giving the time spent in the region $A \subset \mathscr{Q}$ by the path, relative to the time function $\tau$ :

$$
\tau(A)=\tau_{\gamma}(A)=\int_{A} d \tau_{\gamma}(q) .
$$

Let

$$
\mathbb{E}(\tau(A))=\int_{\mathscr{P}} \tau_{\gamma}(A) \mathbb{P}(d \gamma)
$$

10 This seems to involve a new sort of conditional probability analogous to the so-called Palm measure. In particular, it does not appear to be a special case of the conditional distribution relative to a $\Sigma$-algebra or a foliation or given the value of another random variable. That is because any path will involve configurations with many different environments $Y$.

11 When $\Psi_{t}$ is time-dependent, it is natural to suppose that the time-parameter $t$ has physical significance via the changes in typical configurations arising from changes in $\mu^{\Psi_{t}}$. In this situation, treating time as if it were physical and observable seems to be a reasonable approximation. In any case, this is an approximation we almost always make, and it seems to often work quite well. be the expected value with respect to $\mathbb{P}$ of $\tau(A)$ (i.e., the expected time spent by the path in $A$ ) relative to the time function $\tau$. We say that $\mu$ is associated with $\mathbb{P}$ and $\tau$ if

$$
\mu(A)=\mathbb{E}(\tau(A)) .
$$

The measure $\mu$ can also be described as follows. We may identify configuration space $\mathscr{Q}$ with $\mathscr{P} \times \mathbb{R}$ by associating any configuration $q$ with the path to which it belongs and the time along the path:

$$
q \mapsto(\gamma(q), \tau(q))
$$

Under this identification $\mu$ is the product of $\mathbb{P}$ and the Lebesgue measure, $d \mu=d \mathbb{P} d t$.

If $\mu$ is associated with $\mathbb{P}$ and $\tau$, then we have that $\mathbb{P}$ arises from conditioning $\mu$ on $\tau$,

$$
\mathbb{P}(S)=\mu(\check{S} \mid \tau=t)
$$

where $S$ is a set of path and $\check{S}=\{q \in \mathscr{Q} \mid \gamma(q) \in S\}$. Note that the right hand side of 145 does not depend on $t$. (It follows from $(142)$ and $(143)$ that $\mu$ is stationary with respect to the dynamics $T_{s}$ generated by $\tau$ according to (142).) Conversely, when $\mu, \mathbb{P}$, and $\tau$ are related as in (145), $\mu$ is associated with $\mathbb{P}$ and $\tau$.

Suppose now that $\mu$ is stationary for a dynamics $T$ for which $\tau$ is a time function ${ }^{12}$ i.e., such that $\sqrt{142}$ is satisfied. Let $\mathbb{P}$ be a measure on $\mathscr{P}$, the path space of the dynamics. Then the following are equivalent:

$$
\begin{aligned}
& \mathbb{P}(S)=\mu(\{q \in \mathscr{Q} \mid 0 \leq \tau(q) \leq 1 \text { and } \gamma(q) \in S\}) \\
& \mathbb{P}(S)=\mu(\check{S} \mid \tau=t) .
\end{aligned}
$$

When $\mu$ and $\mathbb{P}$ are associated in this way then say that $\mathbb{P}$ is generated by $\mu$ and $T$, and if this is so, then $\mu$ is associated with $\mathbb{P}$ and $\tau$. In particular, if $\tau$ is a time function for any Bohmian dynamics with stationary $\Psi$ (or $\left.\mu^{\Psi}\right)$ then $\mu^{\Psi}$ is associated with $\mathbb{P}^{\Psi}$ and $\tau$, where $\mathbb{P}^{\Psi}$ is the measure generated by $\mu^{\Psi}$ and the Bohmian dynamics.

Furthermore, if the dynamics $T$ is given by a vector field $v, \mathbb{P}$ is generated by $\mu$ and $T$ if and only if

$$
\mathbb{P}(S)=\int_{\Gamma_{S}} J \cdot d \sigma,
$$

where $J=J(\mu, T)$ is the current associated with the dynamics. Here $\Gamma_{S}=\Gamma \cap \breve{S}$, where $\Gamma$ is any cross section of the foliation $\mathscr{P}$, for example the level surface $\tau(q)=t$. If $\mu$ is absolutely continuous with density $\rho$ (with respect to the coordinate measure in some coordinate system), then $J=v \rho$ (with $v$ represented in that coordinate system) and the surface integral above is given by the usual formula.

12 Note that, as a consequence of Poincaré recurrence, in general there might be no time function associated with a dynamics $T$. However, if one does exist, it cannot be unique, because it would depend on the choice of an initial cross section. 


\section{F. A Conditional Probability Formula for Path Space Measures}

In this section and the next we consider a fixed system (and its environment), with configuration $Q=(X, Y)$. We also fix the configuration $Y$ of the environment. We prove a crucial fact for establishing in Sect. VIII G the fundamental conditional probability formula for stationary wave functions, a general fact that we call the path space conditional probability formula.

In this formula we condition on the path space event $\{Y \in \gamma\} \subset \mathscr{P}$ that the path $\gamma$ contains a configuration with environmental configuration $Y$, i.e., on the set of paths $\gamma$ that pass through a configuration with environment $Y$. And in this formula we are interested in the conditional distribution of $X_{Y}$, the configuration of the system when the path passes through the configuration with environment $Y$, a function (random variable) on the subset $\{Y \in \gamma\}$ of path space $\mathscr{P}$.

Path space conditional probability formula: Let $\mathbb{P}$ be a measure on the path space $\mathscr{P}$. Suppose $\mu$ is the measure on the corresponding configuration space $\mathscr{Q}$ associated with $\mathbb{P}$ and a time function $\tau$ that depends only upon the configuration $Y$ of the environment. Then

$$
\mathbb{P}\left(X_{Y} \in d x \mid Y \in \gamma\right)=\mu(X \in d x \mid Y) .
$$

This follows more or less as before (see the second bullet of Sect. VIIIC) with $\mathscr{E}$, as before, corresponding to the configuration of the environment and now $\mathscr{F}$ corresponding to the foliation given by the time function. In more detail, since the time function depends only on $Y, \mathscr{E} \subset \mathscr{F}$. Under the natural identification of path space with any leaf of $\mathscr{F}$, the event $\left\{X_{Y} \in d x\right\}$ becomes $\{X \in d x\}$, the event $\{Y \in \gamma\}$ becomes the event that the environment has configuration $Y$, and by $145 \mathbb{P}$ becomes the appropriate conditional measure.

\section{G. The Fundamental Conditional Probability Formula for Stationary Wave Functions}

We assume now that there exists a time function for the Bohmian dynamics that depends only on the configuration of the environment, an assumption that we call the existence of clock variables for the Bohmian dynam$i c s$. Under this assumption the conditional distribution $\mathbb{P}^{\Psi}\left(X_{Y} \in d x \mid Y \in \gamma\right)$ is given by the usual Born's rule on absolute configuration space.

Fundamental conditional probability formula for stationary wave functions: Suppose that there exists a clock variable for the Bohmian dynamics generated by a stationary wave function $\Psi$ on shape space, as well as one for the Bohmian dynamics in the Schrödinger gauge. Then

$$
\mathbb{P}^{\Psi}\left(X_{Y} \in d x \mid Y \in \gamma\right)=\mu^{\widehat{\Psi}}(\boldsymbol{X} \in d \boldsymbol{x} \mid \boldsymbol{Y})
$$

in any of the first three gauges, ${ }^{13}$ or

$$
\mathbb{P}^{\Psi}\left(X_{Y} \in d x \mid Y \in \gamma\right)=C\left|\widehat{\Psi}_{S}(\boldsymbol{x}, \boldsymbol{Y})\right|^{2} d \boldsymbol{x}
$$

in the Schrödinger gauge (as before, $C$ is a normalization constant) where $\boldsymbol{Y}$ is a lift of $Y$ and $\boldsymbol{x}$ is the lift of $x$ in the frame $\boldsymbol{Y}$. Here $\widehat{\Psi}$ and $\widehat{\Psi}_{S}$ are the lifts of $\Psi$ in any of the first three gauges or in the Schrödinger gauge, respectively.

This is a consequence of the path space probability formula 148). Applying it to the Bohmian dynamics (i) on shape space, (ii) in the first three gauges, and (iii) in the Schrödinger gauge, we obtain the following:

$$
\begin{gathered}
\mathbb{P}^{\Psi}\left(X_{Y} \in d x \mid Y \in \gamma\right)=\mu_{B}^{\Psi}(X \in d x \mid Y) \\
\mathbb{P}^{\widehat{\Psi}}\left(\boldsymbol{X}_{\boldsymbol{Y}} \in d \boldsymbol{x} \mid \boldsymbol{Y} \in \gamma\right)=\mu^{\widehat{\Psi}}(\boldsymbol{X} \in d \boldsymbol{x} \mid \boldsymbol{Y}) \\
\mathbb{P}^{\widehat{\Psi}_{S}}\left(\boldsymbol{X}_{\boldsymbol{Y}} \in d \boldsymbol{x} \mid \boldsymbol{Y} \in \gamma\right)=\mu^{\widehat{\Psi}_{S}}(\boldsymbol{X} \in d \boldsymbol{x} \mid \boldsymbol{Y}),
\end{gathered}
$$

given the existence of clock variables for the Bohmian dynamics. Here $\gamma$ is a path for the dynamics on absolute configuration space.

By 138 the right hand sides of (151) and $(152)$ agree when $\boldsymbol{x}$ and $\boldsymbol{Y}$ are appropriate lifts of $x$ and $Y$, so that (149) follows. Since the currents $J$ associated with $\mu^{\widehat{\Psi}}$ and $\mu^{\Psi_{S}}$ arising from their respective dynamics are the same, it follows that $\mathbb{P}^{\widehat{\Psi}}=\mathbb{P}^{\Psi_{S}}$, so that the right hand sides of 152 and 153 are also the same. Thus 150 follows as well.

\section{H. Typicality}

As a partial summary, we find that on the absolute configuration space level the dynamics and the probabilities for subsystems should be of the usual form. While it is true that on the universal level the connection between $|\Psi|^{2}$ and probability, or, more precisely, typicality, would be broken, this would not be visible in any of the familiar every day applications of quantum mechanics, which are concerned only with subsystems and not with the entire universe.

In particular the patterns described by the quantum equilibrium hypothesis will be typical with respect to a measure, not on absolute configuration space, but on shape space, on the fundamental level, which is fine. There is a widespread misconception with respect to Bohmian mechanics that $|\Psi|^{2}$ for the universe and $|\psi|^{2}$ for subsystems play, physically and conceptually, similar roles. They do not, since the role of $|\Psi|^{2}$ is typicality

\footnotetext{
13 These formulas, for the different gauges, may appear to be incompatible. But for the condition relating $\Psi$ and the time-parameter to hold for both the Schrödinger gauge and any other gauge, the condition on $f$ mentioned above would presumably have to be satisfied, in which case the formulas would agree.
} 
while that of $|\psi|^{2}$ is probability. If this distinction is too subtle, the fact that, from a relational perspective, these objects live on entirely different levels of description, $|\Psi|^{2}$ on the fundamental level, i.e., on shape space, and $|\psi|^{2}$ on absolute configuration space, might make it easier to appreciate how very different they are.

\section{OUTLOOK}

The basic problem in cosmology is to determine which laws govern the universe as a whole. The traditional approach is that of building a story about the universe starting from the physical laws operating at small scales, such as the Standard Model of particle physics, and incorporating them within a theory containing a now missing quantum theory of gravity. However, the relational point of view suggests that there is something basically wrong in treating the universe as a whole as a mere combination of the systems that compose it, say galaxies or cluster of galaxies. We elaborate.

Shape space physics is genuinely holistic, and suggests the holistic character of quantum physics associated with entanglement and quantum nonlocality. To appreciate this point, note that for relational space the state of the universe at a particular location is not, in and of itself, meaningful. In that sense, for shape space physics, there are no local beables, so that locality itself can't easily be meaningfully formulated. Similarly one can't meaningfully consider the behavior of individual particles without reference to other particles, since there is no absolute space in which an individual particle could be regarded as moving. And even for a pair of particles, to speak meaningfully of the distance between them, a third particle would be required, to establish a scale of distance. And similarly for galaxies.

There is one rather conspicuous relational aspect that we've ignored. For indistinguishable particles we should have taken one further quotient and enlarged the similarity group $G$ to include the relevant permutations of particle labels. We believe that this would not be too difficult to do, but have chosen not to do so here.

Quite a bit more difficult is the connection between relational physics and relativistic physics.

- A simple point: In relational physics as discussed here the traditional separation of space and time is retained. While configuration space is replaced by shape space, and time becomes non-metrical, shape space retains an identity separate and distinct from that of (non-metrical) time. This is in obvious contrast with relativistic physics, in which space and time lose their separate identities and are merged into a space-time.

- Simultaneity regained and simultaneity lost: Perhaps the most characteristic feature of relativity is the absence of absolute simultaneity. Not so for relational physics. Since it retains the separation of space and time, an absolute simultaneity is built into the very structure of relational physics as described here. Nonetheless, there is a sense in which simultaneity is lost. As discussed in Sect.VIIID, with relational time the notion of the configuration (or shape) $Q_{t}$ of the universe at "time $t$ " is not physically meaningful. And with what is meaningful - geometrical paths in the space of possible configurations (or shapes) - one can no longer meaningfully compare or ask about the configurations for two different possible histories at the same time. Given the actual configuration of the universe, it is not meaningful to ask about the configuration of an alternative history at that time without further specification of exactly what that should mean.

- Can the relational point of view be merged with or extended to relativity? Can we achieve a relational understanding of space-time? General relativity is certainly a step in that direction, but it does not get us there. Space-time in general relativity is metrical - in a way that neither space nor time are in relational physics. A complete extension, if at all possible, is a real challenge.

- Another possibility: relativity is not fundamental, but-like Newtonian physics in the Newton gauge and quantum physics in the Schrödinger gauge - is, instead, a consequence of a suitable choice of gauge. This possibility, which is suggested by the work of Bryce DeWitt [10] and Barbour and coworkers (see, e.g., 6], [13], and [5]), would be worth carefully exploring.

The discussion in Sect. VIIID, with its focus on geometrical paths as more fundamental from the point of view of relational time (and, more generally, with regard to what is more directly observable), was based on a Bohmian approach to quantum physics. This approach involves a law for the evolution of configurations, yielding geometrical paths, the analysis of which leads ultimately to the Born rule (on absolute space) in a more or less familiar form. Without such an approach - and the paths that it provides - it is not easy to see how one could begin to proceed in a principled manner.

As is well known, non-normalizable wave functions tend to occur in quantum cosmology. Such wave functions would normally be regarded as problematical and unphysical (since the formal structures of orthodox quantum mechanics, with their associated probabilities, are crucially based on the notion of a Hilbert space of squareintegrable, i.e. normalizable, wave functions).

However, for our analysis starting in Sect. VIIID the connection between a measure on path space and a (stationary) non-normalizable measure on configuration space turned out to be crucial. By the very nature of this connection the measure on configuration space and its associated wave function in fact had to be non- 
normalizable. Hence what from an orthodox perspective is a vice is transformed into a virtue in relational Bohmian mechanics.

\section{ACKNOWLEDGMENTS}

We are grateful to Florian Hoffmann for his input to a very early draft of this paper and to Antonio Vassallo for his insights. We thank Sahand Tokasi for stimulating discussions. We thank Eddy Chen and Roderich Tumulka for a careful reading of the manuscript and useful suggestions. The many discussions with Julian Barbour are gratefully acknowledged, especially for sharing with us in his well known enthusiastic way his ideas on shape dynamics. N. Zanghí was supported in part by INFN.

\section{APPENDIX: SOME FACTS ABOUT SECOND-ORDER PARTIAL DIFFERENTIAL OPERATORS}

In local coordinates, any second order partial differential operator (PDO) with real coefficients, self-adjoint with respect to some volume element $\mu(d q)$ is of the form

$$
\mathrm{L}=\sum_{i j} A^{i j} \partial_{i} \partial_{j}+\sum_{i} B^{i} \partial_{i}+C
$$

with symmetric matrix function $A=\left(A^{i j}\right)$, vector $B=$ $\left(B^{i}\right)$, and scalar $C$. So it can be compactly written as

$$
\mathrm{L}=A \nabla \nabla+B \cdot \nabla+C
$$

Note that, while the explicit functions $A=A(q), B=$ $B(q)$ and $C=C(q)$ depend on the coordinate system chosen, $A$ is in fact a tensor, so that the fact that two second order PDOs $L$ and $L^{\prime}$ have equal $A$-parts (pure second-derivative parts) is invariant.

Note that for a Laplace-Beltrami operator $C=0$. Moreover, we shall need the $A$-part of the LaplaceBeltrami operator with respect to the invariant metric $g=f g_{e}$, where $g_{e}$ is the mass-weighted Euclidean metric. According to 68 , we have

$$
A=f^{-1} I
$$

where $I$ is the identity matrix.

Here are some relevant facts: Suppose $L$ and $L^{\prime}$ are second order PDOs on a manifold $M$. If

1. they have the same $A$-part, and

2. are self-adjoint with respect the same measure $\mu$, then they differ by at most a multiplication operator $D=$ $D(q)$, i.e.,

$$
\mathrm{L}^{\prime}=\mathrm{L}+D \text {. }
$$

This is so because their difference, which must be of the form $B \cdot \nabla+D$, must also be self adjoint. Since $D$ is as well, $B \cdot \nabla$ must also be. But for no measure $\mu$ can $B \cdot \nabla$ be self-adjoint on $L^{2}(d \mu)$, unless $B=0$. Moreover, if $\mathrm{L}$ has no $C$-part, then

$$
D=\mathrm{L}^{\prime} 1
$$

where 1 is the constant function equal to 1.
[1] Anderson, E.: The problem of time and quantum cosmology in the relational particle mechanics arena. arXiv preprint arXiv:1111.1472 (2011).

[2] Anderson, E.: The problem of time in quantum gravity. Annalen der Physik, 524, 757-786 (2012).

[3] Barbour, J. B., Bertotti, B.: Mach's principle and the structure of dynamical theories. Proc. Roy. Soc. Lond. A, 382, 295-306 (1982).

[4] Barbour, J.: Scale-invariant gravity: particle dynamics. Classical and Quantum Gravity, 20, 1543 (2003).

[5] Barbour, J.: Shape dynamics. An introduction. In: Quantum Field Theory and Gravity, 257-297. Springer (2012).

[6] Barbour, J., Foster, B. Z., Ó Murchadha, N.: Relativity without relativity. Classical and Quantum Gravity, 19, 3217 (2002).

[7] Bell, J.S.: Speakable and unspeakable in quantum mechanics. Cambridge University Press (1987).

[8] Bohm, D.: A suggested interpretation of the quantum theory in terms of" hidden" variables. I. Physical Review, 85, 166 (1952).

[9] Bricmont, J.: Making sense of quantum mechanics. Springer Science \& Business Media (2016).
[10] DeWitt, B. S.: Spacetime as a sheaf of geodesics in superspace. In: Relativity, 359-374, Springer, (1970).

[11] Dürr, D., Goldstein, S., Zanghì, N.: Quantum equilibrium and the origin of absolute uncertainty. Journal of Statistical Physics, 67, 843-907 (1992).

[12] Dürr, D., Goldstein, S., Zanghì, N.: Quantum physics without quantum philosophy. Springer Science \& Business Media (2012).

[13] Gomes, H., Gryb S., Koslowski, T.: Einstein gravity as a 3d conformally invariant theory. Classical and Quantum Gravity, 28, 045005 (2011).

[14] Gryb, S.: Jacobi's principle and the disappearance of time. Phys. Rev. D, 81, 044035 (2010).

[15] Kendall, D.G.:. The diffusion of shape. Advances in applied probability, 9, 428-430 (1977).

[16] Kendall, D.G., Barden, D., Carne, T.K. Le, H.: Shape and shape theory, John Wiley \& Sons (2009).

[17] Koslowski, T.: Quantum inflation of classical shapes. Foundations of Physics, 47, 625-639 (2017).

[18] Le, H., Kendall, D.G.: The Riemannian structure of Euclidean shape spaces: a novel environment for statistics. The Annals of Statistics, 21, 1225-1271 (1993).

[19] Codá Marques, F.: Scalar curvature, conformal geom- 
etry, and the Ricci flow with surgery. In: Proceedings of the International Congress of Mathematicians 2010, 811-829. World Scientific (2010).

[20] Mercati, F.: Shape dynamics: relativity and relationalism. Oxford University Press (2018).

[21] Small, C.G.: The statistical theory of shape. Springer
Science \& Business Media (2012).

[22] Vassallo, A., Ip, P.H.: On the conceptual issues surrounding the notion of relational Bohmian dynamics. Foundations of Physics, 46, 943-972 (2016). 